IJMMS 32:9 (2002) 515-554

PII. S016117120201181X

http://ijmms.hindawi.com

(c) Hindawi Publishing Corp.

\title{
SKEIN MODULES OF LINKS IN CYLINDERS OVER SURFACES
}

\author{
JENS LIEBERUM
}

Received 3 February 2001 and in revised form 18 December 2001

We define the Conway skein module $\mathscr{C}(M)$ of ordered based links in a 3-manifold $M$. This module gives rise to $\mathscr{C}(M)$-valued invariants of usual links in $M$. We determine a basis of the $\mathbb{Z}[z]$-module $\mathscr{C}(\Sigma \times[0,1]) / \operatorname{Tor}(\mathscr{C}(\Sigma \times[0,1]))$, where $\Sigma$ is the real projective plane or a surface with boundary. For cylinders over the Möbius strip or the projective plane, we derive special properties of the Conway skein module, among them a refinement of a theorem of Hartley and Kawauchi about the Conway polynomial of strongly positive amphicheiral knots in $S^{3}$. In addition, we determine the Homfly and Kauffman skein modules of $\Sigma \times[0,1]$ where $\Sigma$ is an oriented surface with boundary.

2000 Mathematics Subject Classification: 57M25.

1. Introduction. In 1969, Conway found a normalized version $\tilde{\nabla}$ of the Alexander polynomial of a link. It satisfies a so-called skein relation on certain triples of links differing only by local modifications, namely,

$$
\tilde{\nabla}(\swarrow \searrow)-\tilde{\nabla}(\swarrow \searrow)=z \tilde{\nabla}(\swarrow \unlhd)
$$

(see $[1,7])$. The Conway polynomial also satisfies $\tilde{\nabla}\left(O_{n}\right)=\delta_{1 n}$, where $O_{n}$ is the trivial link with $n$ components and $\delta_{i j}$ is 1 if $i=j$, and 0 otherwise. The skein relation together with $\tilde{\nabla}\left(O_{1}\right)=1$ uniquely determine the isotopy invariant $\tilde{\nabla}(L)$ for every link $L$. More precisely, the so-called skein module $\mathscr{C}\left(S^{3}\right)$, generated over $\mathbb{Z}\left[z^{ \pm 1}\right]$ by isotopy classes of links modulo the skein relation, is free with basis $O_{1}$. The Jones, Homfly, and Kauffman polynomials of links in $S^{3}[8,10,11]$ can be characterized by similar skein relations. In 1988, Przytycki and Turaev generalized the Homfly and Kauffman polynomials to links in a solid torus and determined the corresponding skein modules. Since then, various versions of skein modules related to these polynomials have been studied [19], among them the Homfly skein module of links in a handlebody [18].

In 1990, Vassiliev invariants were introduced [23]. They can be characterized as follows: every link invariant $v$ with values in an abelian group can recursively be extended to an invariant of singular links by $v(\searrow)=v(\swarrow)-v(\searrow)$. Vassiliev invariants $v$ of degree $n$ of links are those invariants that vanish on singular links with $n+1$ double points. If the 3-manifold is not orientable, then there exists no preferred choice of a sign in $v(\swarrow)= \pm v(\swarrow) \mp v(\searrow)$, but it still makes sense to speak of Vassiliev invariants of degree $n$. Let $I=[0,1]$. In 1996, Andersen, Mattes, and Reshetikhin [2] found a universal Vassiliev invariant $Z_{\Sigma \times I}$ of links in a cylinder over an orientable surface $\Sigma$ with boundary. We use $Z_{\Sigma \times I}$ to determine the Homfly and Kauffman skein modules of $\Sigma \times I$. In comparison with [18], our choice of a basis of the Homfly skein module 
has some technical advantages. The result seems to be new for the Kauffman skein module.

In [15], the invariant $Z_{\Sigma \times I}$ was extended to a universal Vassiliev invariant of links in cylinders over nonorientable surfaces with boundary and over the real projective plane $P^{2}$. The main idea that led to this paper was to extract an explicitly computable link invariant from $Z_{\Sigma \times I}$ in the nonorientable case. We define a skein module $\mathscr{C}(M)$ of a 3-manifold $M$ using a version of the skein relation in (1.1) for ordered based links. In Theorem 4.1, we establish an isomorphism of $\mathbb{Z}[z]$-modules,

$$
\mathscr{C}^{\#}(\Sigma \times I) \cong \mathbb{Z}[z] \otimes_{\mathbb{Z}} \mathscr{C}_{0}^{\#}(\Sigma \times I)
$$

where $\Sigma=P^{2}$ or $\Sigma$ is a nonorientable surface with boundary, $\mathscr{C}^{\#}(M)$ is the quotient of the $\mathbb{Z}[z]$-module $\mathscr{C}(M)$ by its torsion submodule, and $\mathscr{C}_{0}^{\#}(M)$ is the quotient of the $\mathbb{Z}$-module $\mathscr{C}(M) /(z \mathscr{C}(M))$ by its torsion submodule. We determine the structure of $\mathscr{C}_{0}^{\#}(M)$ for an arbitrary 3-manifold. It is isomorphic to a tensor product of a polynomial algebra and an exterior algebra. A basis of $\mathfrak{C}^{\#}(\Sigma \times I)$ is given by certain descending links. We describe explicitly how to compute a Conway polynomial inducing the isomorphism in (1.2) and how to obtain invariants of links (without order or basepoints) from the Conway skein module. When $\Sigma$ is the Möbius strip $X$ or $\Sigma=P^{2}$ we show that the isomorphism in (1.2) is an isomorphism of involutive algebras (Theorem 4.2). In addition, we have the following theorem for the Conway polynomials $\tilde{\nabla}$ of knots in $P^{2} \times I$ and in $S^{2} \times I$.

THEOREM 1.1. For a noncontractible knot $K \subset P^{2} \times I$

$$
\tilde{\nabla}\left(p^{-1}(K)\right)=\tilde{\nabla}(K)^{2},
$$

where $p: S^{2} \times I \rightarrow P^{2} \times I$ denotes the 2-fold covering map.

See Theorem 4.3 for an equivalent formulation of Theorem 1.1. For strongly positive amphicheiral knots in $S^{3}$, a theorem of Hartley and Kawauchi [9] says that the Conway polynomial is a square. Theorem 1.1 is a refinement of this result (see Corollary 4.4) because it assures that the square root of the Conway polynomial of $p^{-1}(K)$ can be computed directly using the Conway skein relation for ordered based links in $P^{2} \times I$.

The paper is organized as follows. In Section 2, we define $\mathscr{C}(M)$ and prove some of its general properties. After this section we concentrate on the case $M=\Sigma \times I$. In Section 3, we introduce decomposed surfaces and make a choice of representatives of conjugacy classes in the fundamental group of $\Sigma$. This choice is important in the definition of a descending link. Only a careful choice leads to a basis of $\mathfrak{C}^{\#}(\Sigma \times I)$ over $\mathbb{Z}[z]$. In Section 4, we state Theorems 4.1, 4.2, and 4.3. Sections 5 to 9 are devoted to the proof of these theorems. In Section 5, we show that descending knots and the socalled cabled descending knots generate $\mathscr{C}(\Sigma \times I)$ as an algebra. Making computations in $\mathscr{C}^{\#}(X \times I)$, we show in Section 6 that cabled descending knots vanish in $\mathscr{C}^{\#}(\Sigma \times I)$. In Section 7, we introduce labeled ordered based chord diagrams and prove that a map $W_{\mathfrak{g} \rho_{0}}^{\text {ob }}$ defined on these diagrams is compatible with certain relations. In Section 8, we compose the universal Vassiliev invariant $Z_{\Sigma \times I}$ adapted to ordered based links with $W_{\mathfrak{g} \int_{0}}^{\mathrm{ob}}$ to prove Theorems 4.1 and 4.2. In Section 9, we prove Theorem 4.3 using a 
map between chord diagrams induced by the covering $S^{2} \times I \rightarrow P^{2} \times I$. In an appendix, we determine the structure of the Homfly and Kauffman skein modules of $\Sigma \times I$ for oriented surfaces $\Sigma$ with boundary.

2. The Conway skein module. Throughout this paper, we work in the PL-category. Let $M$ be a connected 3-manifold equipped with an oriented subset $U$ homeomorphic to a closed 3-ball $D^{3}$. We denote the fundamental group of $M$ with respect to a basepoint in $U$ by $\pi_{1}(M)$. The first Stiefel-Whitney class induces a homomorphism $\sigma: \pi_{1}(M) \rightarrow \mathbb{Z} / 2$ called orientation character of $M$. Explicitly, if a local orientation at the basepoint stays the same when we push it around $w \in \pi_{1}(M)$, then we have $\sigma(w)=0$, and $\sigma(w)=1$ otherwise.

Ordered based links in $M$ are links in $M$ with a linear order of their components and with a basepoint in $U$ on each component. In particular, every component of an ordered based link meets $U$. The basepoints are not allowed to leave $U$ during an isotopy (resp., homotopy) of ordered based links. The homotopy class of a based knot $K$ can canonically be considered as an element $\alpha(K) \in \pi_{1}(M)$. We denote $\sigma(\alpha(K))$ simply by $\sigma(K)$. Notice that $\sigma(K)$ does not depend on the basepoint of $K$. We will regard the empty link $\varnothing$ as an ordered based link because this will simplify slightly the statement of our results.

In Figure 2.1 parts of ordered based links are shown. Numbers 1 and 2 near the basepoints in this figure indicate that the corresponding component is first or second in the order of this link.

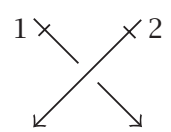

$L_{+}$

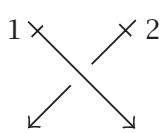

$L_{-}$

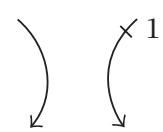

$L_{\|}$

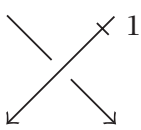

$L_{+}$

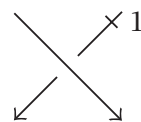

$L_{-}$

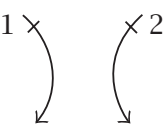

$L_{\|}$

FIGURE 2.1. Skein triples of ordered based links.

Ordered based links $\left(L_{+}, L_{-}, L_{\|}\right)$form a skein triple if they differ inside of a ball $D^{3} \subset U$ as one of the two triples $\left(L_{+}, L_{-}, L_{\|}\right)$in Figure 2.1, they coincide on the outside of this ball, and the order and basepoints of the remaining components also coincide. We say that $L_{+}$and $L_{-}$are related by changing a crossing and $L_{\|}$is obtained from $L_{+}$ or $L_{-}$by splicing a crossing.

DEFINITION 2.1. Let $\mathscr{C}(M)$ be the $\mathbb{Z}[z]$-module generated by isotopy classes of ordered based links and the following relations (Ord), (Bas), and (Skein):

(Ord): $L=(-1)^{\sigma\left(K_{1}\right) \sigma\left(K_{2}\right)} L^{\prime}$, where $K_{1}, K_{2}$ are two components of an arbitrary ordered based link $L$ that are neighbors in the order of the components of $L$. The ordered based link $L^{\prime}$ is equal to $L$ except that the order of $K_{1}$ and $K_{2}$ is interchanged.

(Bas): $L=(-1)^{\sigma(a) \sigma(b)} L^{\prime}$, where $L$ and $L^{\prime}$ are ordered based links that are equal except for one basepoint on a component $K$ of $L$ and $K^{\prime}$ of $L^{\prime}$. The element $a \in \pi_{1}(\Sigma \times I)$ 
is given by the curve described by the basepoint of $K$ when it is pushed along $K$ until it coincides with the basepoint of $K^{\prime}$, and $b=\alpha\left(K^{\prime}\right) a^{-1}$.

(Skein): $L_{+}-L_{-}=z L_{\|}$, where $\left(L_{+}, L_{-}, L_{\|}\right)$is a skein triple of ordered based links.

Let the homomorphism of rings $\varphi: \mathbb{Z}[z] \rightarrow \mathbb{Z}[z]$ be given by $\varphi(p)(z)=p(-z)$. We turn $\mathscr{b}$ into a functor in the sense of the following proposition.

Proposition 2.2. Let the 3-manifolds $M_{i}(i=1,2)$ be equipped with the oriented subsets $U_{i} \cong D^{3}$, and let $i: M_{1} \rightarrow M_{2}$ be an embedding with $i\left(U_{1}\right) \subseteq U_{2}$.

(1) If the orientations of $i\left(U_{1}\right)$ and $U_{2}$ coincide, then $i$ induces a morphism of $\mathbb{Z}[z]$ modules $i_{*}: \mathscr{C}\left(M_{1}\right) \rightarrow \mathscr{C}\left(M_{2}\right)$.

(2) If the orientations of $i\left(U_{1}\right)$ and $U_{2}$ are opposite, then $i$ induces a morphism of $\mathbb{Z}$-modules $i_{*}: \mathscr{C}\left(M_{1}\right) \rightarrow \mathscr{C}\left(M_{2}\right)$ satisfying $i_{*}(p m)=\varphi(p) i_{*}(m)$ for all $p \in \mathbb{Z}[z]$ and $m \in \mathscr{b}\left(M_{1}\right)$.

SKETCH OF PROOF. (1) Is obvious.

(2) The only defining relation of $\mathscr{C}\left(M_{1}\right)$ that depends on the orientation of $U_{1}$ is the skein relation. Reversing the orientation of $U_{1}$ interchanges the parts $L_{+}$and $L_{-}$of a link which can be compensated by replacing $z$ by $-z$. In this way we obtain part (2) of the proposition using part (1).

The existence of a suitable homeomorphism from $M$ to itself implies the following corollary, which explains why we do not refer explicitly to $U$ in the name of $\mathscr{C}(M)$.

COROLLARY 2.3. If the 3-manifold $M$ is oriented (resp., nonorientable), then for different choices of $U_{i} \subset M_{i}=M$ with $U_{i} \cong D^{3}$ equipped with the induced (resp., an arbitrary) orientation the $\mathbb{Z}[z]$-modules $\mathscr{C}\left(M_{i}\right)$ are isomorphic.

Let $c$ be an arbitrary crossing of an ordered based link. We apply an isotopy such that $c$ is contained in $U$. Then we use relations (Ord) and (Bas) such that $c$ becomes a crossing involved in a skein triple. The skein relation then implies that the $\mathbb{Z}$-module $\mathscr{C}_{0}(M)=\mathscr{C}(M) /(z \mathscr{C}(M))$ is generated by homotopy classes of ordered based links. We turn a disjoint union of ordered based links $L=L_{1} \cup L_{2}$ into an ordered based link by extending the order of the components of $L_{i}$ to $L$ by $K_{1}<K_{2}$ for based knots $K_{i} \subset L_{i}$. This operation is not well defined on $\mathscr{C}(M)$, but it turns $\mathscr{C}_{0}(M)$ into an associative ring with 1-element $\varnothing$. Let $T=\operatorname{Tor}\left(\mathscr{C}_{0}(M)\right)$ be the torsion submodule of the $\mathbb{Z}$-module $\mathscr{C}_{0}(M)$ which is a 2-sided ideal of $\mathscr{C}_{0}(M)$. Define $\mathscr{C}_{0}^{\#}(M)=\mathscr{C}_{0}(M) / T$. We describe the structure of $\mathscr{C}_{0}^{\#}(M)$. Denote the conjugacy class of an element $w \in \pi_{1}(M)$ by $[w]$. Let

$$
\begin{aligned}
& \mathscr{Y}_{1}=\left\{[w] \mid w \in \pi_{1}(M), \sigma(w)=1\right\}, \\
& \mathscr{S}_{0}^{*}=\left\{[w] \mid w \in \pi_{1}(M), \sigma(w)=0 \text { and } N(w) \subseteq \operatorname{Ker} \sigma\right\},
\end{aligned}
$$

where $N(w)=\left\{v \in \pi_{1}(M) \mid v w=w v\right\}$ is the normalizer of $w$. For a set $X$, let $V_{X}$ be the free $\mathbb{Z}$-module with basis $\left\{t_{x} \mid x \in X\right\}$. Denote the symmetric algebra on a free $\mathbb{Z}$-module $V$ by $S(V)$ and the exterior algebra by $\Lambda(V)$. For $w \in \pi_{1}(M)$ we denote the corresponding based knot in $\mathscr{C}_{0}^{\#}(M)$ by $\bar{K}_{w}$. 
Proposition 2.4. For every choice $\hat{\varphi}_{0}^{*}$ of representatives of the conjugacy classes in $\mathscr{S}_{0}^{*}$ exists an isomorphism of algebras

$$
\psi: S\left(V_{\hat{S}_{0}^{*}}\right) \otimes \Lambda\left(V_{\mathscr{S}_{1}}\right) \longrightarrow \mathscr{C}_{0}^{\#}(M)
$$

given by mapping $t_{v} \otimes 1$ to $\bar{K}_{v}\left(v \in \hat{\mathscr{Y}}_{0}^{*}\right)$ and $1 \otimes t_{[w]}$ to $\bar{K}_{v}$ for some $v \in[w] \in \mathscr{Y}_{1}$.

Proof. The $\mathbb{Z}$-module $\mathscr{C}_{0}(M)$ can be described by noncommutative generators $k_{w}$ $\left(w \in \pi_{1}(M)\right)$ and the relations

$$
\begin{aligned}
\left(\operatorname{Ord}^{\prime}\right): k_{v} k_{w} & =(-1)^{\sigma(v) \sigma(w)} k_{w} k_{v}, \\
\left(\operatorname{Bas}^{\prime}\right): k_{v w} & =(-1)^{\sigma(v) \sigma(w)} k_{w v},
\end{aligned}
$$

for all $v, w \in \pi_{1}(M)$. By the relation (Bas'), we have $k_{a}=k_{b}$ for all $a, b \in[a] \in \mathscr{S}_{1}$. Relation $\left(\mathrm{Ord}^{\prime}\right)$ implies that $k_{a}^{2}=-k_{a}^{2} \in \mathscr{C}_{0}(M)$. Therefore, we have $k_{a}^{2}=0 \in \mathscr{C}_{0}^{\#}(M)$. Now relation $\left(\mathrm{Ord}^{\prime}\right)$ implies that $\psi$ is well defined. If $\sigma(w)=0$ and there exists $v \in$ $\pi_{1}(M)$ with $v w=w v$ and $\sigma(v)=1$, then

$$
k_{w}=k_{v w v^{-1}}=(-1)^{\sigma(v) \sigma\left(w v^{-1}\right)} k_{w v^{-1} v}=-k_{w},
$$

implying that $k_{w}=0 \in \mathscr{C}_{0}^{\#}(M)$. Therefore, $\psi$ is surjective. Since we know a presentation of $\mathscr{C}_{0}(M)$ by generators and relations, it is easy to verify that an inverse map of $\psi$ is well defined.

Now we fix a choice of a linearly ordered set $\hat{\mathscr{S}}^{*}$ consisting of representatives of $\mathscr{Y}_{0}^{*} \cup \mathscr{Y}_{1}$. Then by Proposition 2.4 we can equip $\mathscr{C}_{0}^{\#}(M)$ with the basis

$$
\bar{K}_{w_{1}} \cdots \bar{K}_{w_{n}}, \quad \text { where } w_{i} \in \hat{\mathscr{Y}}^{*}, w_{i} \leq w_{i+1},\left(w_{i}=w_{i+1} \Longrightarrow \sigma\left(w_{i}\right)=0\right) .
$$

We define the linear map $\theta: \mathscr{C}_{0}^{\#}(M) \rightarrow \mathbb{Z}$ to be equal to 1 on these basis elements. For nonorientable $M$, the definition of $\theta$ depends on the representatives chosen for $\mathscr{S}_{0}^{*}$ and on the order chosen on $\mathscr{S}_{1}$.

The module $\mathscr{C}(M)$ gives rise to invariants of links in $M$ (without order or basepoints) as follows. Given a link $L$, we choose a basepoint $b_{i}$ on each component and pull all basepoints into $U$ along disjoint paths $\beta_{i}$. Then we choose an arbitrary order of the components of $L$ and obtain an ordered based link $L_{b}$. We denote the image of $L_{b} \in \mathscr{C}(M)$ under the $\mathbb{Z}$-linear projection $\mathscr{C}(M) \rightarrow \mathscr{C}_{0}^{\#}(M)$ by $\bar{L}_{b}$. Define $v_{i}(L)$ and $C(L)$ by

$$
\begin{gathered}
\frac{\mathscr{C}(M)}{z^{i+1 \mathscr{C}(M)}} \ni v_{i}(L)=\theta\left(\bar{L}_{b}\right) \bar{L}_{b}, \\
\{\{a, b\} \mid a, b \in \mathscr{C}(M)\} \ni C(L)= \begin{cases}\left\{\theta\left(\bar{L}_{b}\right) \bar{L}_{b}\right\}, & \text { if } \theta\left(\bar{L}_{b}\right) \neq 0, \\
\left\{ \pm \bar{L}_{b}\right\}, & \text { if } \theta\left(\bar{L}_{b}\right)=0 .\end{cases}
\end{gathered}
$$

Proposition 2.5. The maps $C$ and $v_{i}(i \geq 0)$ are isotopy invariants of links. In addition, the maps $v_{i}$ are Vassiliev invariants of degree $i$.

Proof. Let $L$ be a link and let $L_{a}, L_{b}$ be ordered based links obtained from $L$ by choosing basepoints $a_{i}$ and $b_{i}$ on the components of $L$ that are pulled into $U$ along the 
paths $\alpha_{i}$ and $\beta_{i}$, respectively. We first assume that all the paths $\alpha_{i}$ and $\beta_{i}$ are disjoint. Then we can pull the points $b_{i}$ on $L_{a}$ into $U$ along the $\beta_{i}$, and the points $a_{i}$ on $L_{b}$ into $U$ along $\alpha_{i}$. This shows that we can pass from $L_{a}$ to $L_{b}$ by isotopies of ordered based links and by application of relations (Ord) and (Bas). The relations (Ord) and (Bas) influence only the signs, and $\theta\left(\bar{L}_{b}\right)$ changes signs simultaneously with $\bar{L}_{b}$. If the paths $\alpha_{i}$ and $\beta_{i}$ are not disjoint, then we choose the basepoints $c_{i}$ of $L$ and paths $\gamma_{i}$ disjoint to $\alpha_{i}$ and $\beta_{i}$ and apply the argument above to the pairs $\left(L_{a}, L_{c}\right)$ and $\left(L_{c}, L_{b}\right)$. This shows that the maps $v_{i}$ and $C$ are well defined isotopy invariants of links. It follows from the skein relation by the same arguments as for the usual Conway polynomial of links that the maps $v_{i}$ are Vassiliev invariants of degree $i$ (see [24]).

3. Decomposed surfaces. Now we consider the special case $M=\Sigma \times I$, where $\Sigma$ is a connected compact surface and $I=[0,1]$ is an oriented interval. First assume that $\partial \Sigma \neq \varnothing$. We choose an oriented subset $B_{0} \cong D^{2}$ of $\Sigma$ such that $B_{0} \cap \partial \Sigma \neq \varnothing$. Define $\mathscr{C}(\Sigma \times I)$ with respect to $U=B_{0} \times I \cong D^{3}$. Fix the choice of a point $u \in B_{0} \cap \partial \Sigma$. For the following definition we need some notation: we denote the fundamental group of $\Sigma$ with respect to a basepoint in $B_{0}$ by $\pi_{1}(\Sigma)$. Let $p_{\Sigma}$ and $p_{I}$ be the projections from $\Sigma \times I$ to $\Sigma$ and $I$, respectively. For $x \in \sum \times I$ we call the value $p_{I}(x)$ the height of $x$.

DEFINITION 3.1. (1) A based knot $K \subset \Sigma \times I$ is called descending with respect to its basepoint, if the height of $K$ is descending when we travel along $K$ starting at the basepoint $A$ and following the orientation of $K$ until we reach a point $A^{\prime}$ with $p_{\Sigma}(A)=p_{\Sigma}\left(A^{\prime}\right)$ from which $K$ leads back to $A$ by increasing the height and by keeping the projection to $\Sigma$ constant.

(2) A based knot $K \subset \sum \times I$ is called a descending knot, if $K$ is descending with respect to its basepoint $A$ and there exists a neighborhood $V \cong D^{2}$ of $u$ in $B_{0}$ such that $(V \times I) \cap K$ consists of an interval containing $A$.

The important property of descending knots is that for $w \in \pi_{1}(\Sigma)$ there exists up to isotopy exactly one descending knot $K_{w}$ such that $\alpha\left(K_{w}\right)=w$. If $v, w \in \pi_{1}(\Sigma)$ are conjugate then we can pass from $K_{v}$ to $K_{w}$ by isotopies and crossing changes and by moving the basepoint. In this section we choose a set $\hat{\mathscr{S}}$ of representatives of conjugacy classes in $\pi_{1}(\Sigma)$ with good properties (compare the example preceding (5.1) for a choice with bad properties).

DEFINITION 3.2. A decomposed surface is a triple $\left(\Sigma,\left(B_{0}, B_{1}, \ldots, B_{k}\right), u\right)$ where $\Sigma$ is a surface, $I^{2} \cong B_{0} \subset \Sigma$ is oriented, $u \in B_{0} \cap \partial \Sigma$, and $I^{2} \cong B_{i} \subset \Sigma$ such that $\bigcup_{i=0}^{k} B_{i}=\Sigma$ and conditions (3.1) are satisfied,

$$
\begin{aligned}
& B_{0} \cap B_{i} \cong I \times\{0,1\} \quad \text { for } i \geq 1, \\
& B_{i} \cap B_{j}=\varnothing \quad \text { for } i \neq j, i, j \geq 1 .
\end{aligned}
$$

We represent decomposed surfaces graphically as shown in Figure 3.1 by an example. By convention, the orientation of $B_{0}$ is counterclockwise in this figure. We also represent $\Sigma \times I$ as in Figure 3.1 where we assume that on $B_{0}$ the interval $I$ directs towards the reader. 


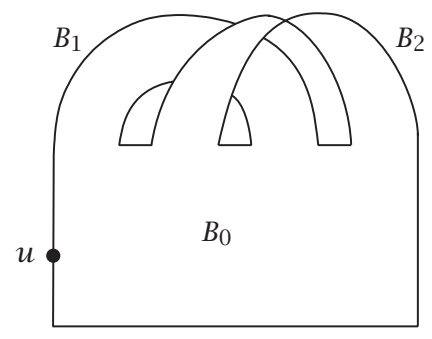

FigURE 3.1. A picture of a decomposed surface.

The decomposition of $\Sigma$ determines generators $x_{i}(i=1, \ldots, k)$ of $\pi_{1}(\Sigma)$ that pass through the band $B_{i}$ exactly once in the clockwise sense in our pictures and that do not meet the other bands $B_{j}(j \geq 1, i \neq j)$. For $w \in \pi_{1}(\Sigma)$ we denote by $\ell(w)$ the length of the unique reduced expression of $w$ in the generators $x_{i}$ of the free group $\pi_{1}(\Sigma)$ and we denote the generator at position $i(1 \leq i \leq \ell(w))$ in this expression by $w(i)$. Traveling along $\partial B_{0}$ in the clockwise sense starting at $u$, we number the $2 k$ intervals $B_{i} \cap B_{0}(i=1, \ldots, k)$ from 1 to $2 k$. We denote these intervals by $I_{v}(v \in\{1, \ldots, 2 k\})$. This numbering determines maps $s, d: \pi_{1}(\Sigma) \rightarrow\{1, \ldots, 2 k\}$ where $I_{s(w)}$ (resp., $\left.I_{d(w)}\right)$ is the interval that a generic representative of $w$ intersects first (resp., last). For example, we have $s\left(x_{1}^{-1} x_{2}\right)=3$ and $d\left(x_{1}^{-1} x_{2}\right)=4$ for the decomposed surface in Figure 3.1. We define an order on elements of $\pi_{1}(\Sigma)$ of fixed length $r$ such that for $v, w$ with $s(v)<s(w)$ we have $v<w$. For the remaining pairs $(v, w) \in \pi_{1}(\Sigma)^{2}$ with $v \neq w$ we choose $m>0$ such that $t:=v(1) \cdots v(m-1)=w(1) \cdots w(m-1)$ and $x:=v(m) \neq$ $w(m)=: y$ and say that $v<w$ if

$$
\sigma(t)+\rho(s(y), s(x))+\rho(d(t), s(x))+\rho(d(t), s(y)) \equiv 0 \bmod 2,
$$

where $\rho(a, b)$ is 0 for $a>b$ and 1 for $a \leq b$. Notice that we have $s(x) \neq s(y), d(t) \neq$ $s(x), d(t) \neq s(y)$. See Figure 3.2 for an example.

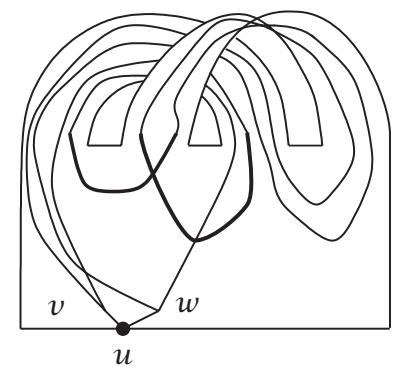

$$
\begin{aligned}
& m=3 \\
& x=x_{1}^{-1}, y=x_{1}, t=x_{1} x_{2}^{-1} \\
& s(x)=3, s(y)=1, d(t)=2 \\
& \sigma(t)+\rho(1,3)+\rho(2,3)+\rho(2,1)= \\
& 1+1+1+0 \equiv 1 \bmod 2
\end{aligned}
$$

FiguRE 3.2. $x_{1} x_{2}^{-1} x_{1}^{-1}=v>w=x_{1} x_{2}^{-1} x_{1}$.

We can visualize the relation defined above as follows: the condition $s(v)<s(w)$ says that if the first interval where $v$ enters a band lies to the left of the first interval where $w$ enters a band, then we have $v<w$. For the second condition, represent $v$ by a generic loop on $\Sigma$. We start drawing $w$ by entering the first band to the right of 

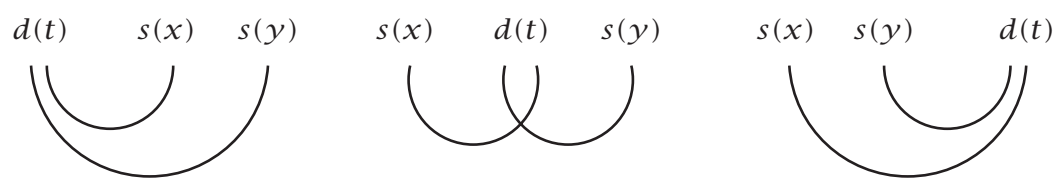

FIGURE 3.3. Configurations of $(s(x), s(y), d(t))$ and intersections.

$v$ and then by running close and parallel to $v$ through the first $m-1$ bands. Then $w$ leaves the $m-1$ st band to the left of $v$ if and only if $\sigma(t)=0$. Three configurations of $(s(x), s(y), d(t)) \in \mathbb{N}^{3}$ and the possible ways to continue drawing $w$ for $\sigma(t)=0$ are shown in Figure 3.3.

The remaining possibilities in Figure 3.3 for $\sigma(t)=0$ are given by interchanging $x$ and $y$. The case $\sigma(t)=1$ is treated in a similar way. We see that

$$
\sigma(t)+\rho(s(y), s(x))+\rho(d(t), s(x))+\rho(d(t), s(y)) \equiv 1 \bmod 2
$$

if and only if the part of $w$ corresponding to $y$ going from the interval $d(t)$ to $s(y)$ must intersect the part of $v$ corresponding to $x$ going from $d(t)$ to $s(x)$. It is easy to verify that the defined relation is an order relation.

An important property of $v, w \in \pi_{1}(\Sigma)$ with $v>w$ is the following. Assume that $v$ and $w$ are represented by generic loops on $\Sigma$ corresponding to reduced words, we have $v(1)=w(1), v$ enters the first band at the interval $s(v)$ to the left of $w$, and $v$ and $w$ have no intersections while they are traversing the bands $B_{i}(i \geq 1)$. Choose the smallest number $n$ such that the part of $v$ going from the interval $d(v(n-1))$ to $s(v(n))$ intersects the part of $w$ going from $d(w(n-1))$ to $s(w(n))$ in an odd number of points. Then we necessarily have $v(i)=w(i)$ for all $i<n$.

Now we can choose representatives $\hat{w}$ of conjugacy classes of elements $w \in \pi_{1}(\Sigma)$ as follows:

$$
\begin{aligned}
& \dot{w}=\min \left\{v \mid v \text { is cyclically reduced, }[v]=[w] \text { or }\left[v^{-1}\right]=[w]\right\}, \\
& \hat{w}= \begin{cases}\dot{w} & \text { if }[\dot{w}]=[w], \\
\dot{w}^{-1} & \text { if }\left[\dot{w}^{-1}\right]=[w] .\end{cases}
\end{aligned}
$$

For a decomposed surface we choose $\hat{\mathscr{S}}:=\left\{\hat{w} \mid w \in \pi_{1}(\Sigma)\right\}$. For $\Sigma=P^{2}$ we obviously have to choose $\hat{\mathscr{S}}=\pi_{1}\left(P^{2}, *\right) \cong \mathbb{Z} / 2$. By a descending knot $K$ we mean, in this case, a based knot $K$ with constant height $p_{I}(K)$. The isotopy class of a descending knot $K$ is again uniquely determined by $\alpha(K) \in \pi_{1}\left(P^{2}, *\right)$.

For $i=0,1$, denote by $f_{i}$ an orientation preserving homeomorphism $I \cong$ $[i / 2,(i+1) / 2]$. By Proposition 2.2 we obtain isomorphisms of $\mathbb{Z}[z]$-modules,

$$
\left(\operatorname{id} \times f_{i}\right)_{*}: \mathscr{b}(\Sigma \times I) \rightarrow \mathscr{b}\left(\sum \times\left[\frac{i}{2}, \frac{(i+1)}{2}\right]\right) .
$$

The identity $\Sigma \times[1 / 2,1] \cup \Sigma \times[0,1 / 2]=\Sigma \times I$ induces a $\mathbb{Z}[z]$-bilinear product,

$$
\mathscr{C}\left(\sum \times I\right) \times \mathscr{b}\left(\sum \times I\right) \cong \mathscr{C}\left(\sum \times\left[\frac{1}{2}, 1\right]\right) \times \mathscr{C}\left(\sum \times\left[0, \frac{1}{2}\right]\right) \rightarrow \mathscr{C}\left(\sum \times I\right),
$$


where we extend the order of the components of ordered based links $L_{1}, L_{2}$ to their product $L_{1} L_{2}$ by $K_{1}<K_{2}$ for $K_{1} \subseteq L_{1}, K_{2} \subseteq L_{2}$. The projection $\mathscr{C}\left(\sum \times I\right) \rightarrow \mathscr{C}_{0}\left(\sum \times I\right)$ becomes a homomorphism of rings.

Since the multiplication in $\mathscr{C}(\Sigma \times I)$ is in general not commutative, we use a standard order for products of descending knots. For this purpose we fix an arbitrary choice of an order on the set $\hat{\mathscr{Y}}$. For example, for a decomposed surface we can take the lexicographical order on the alphabet $x_{1}, x_{1}^{-1}, x_{2}, \ldots$, using reduced expressions.

DEFinition 3.3. An $\hat{\mathscr{9}}$-descending link is an ordered based link isotopic to a product of descending knots $K_{1} \cdots K_{n}$ with $\alpha\left(K_{i}\right) \in \hat{\mathscr{Y}}$ and $\alpha\left(K_{1}\right) \leq \cdots \leq \alpha\left(K_{n}\right)$.

4. Main results about the Conway skein module. Let $\operatorname{Tor}(\mathscr{C}(M))=\{m \in \mathscr{C}(M) \mid$ $\exists p \in \mathbb{Z}[z]: p m=0\}$ be the torsion submodule of $\mathscr{C}(M)$ and denote the quotient $\mathscr{C}(M) / \operatorname{Tor}(\mathscr{C}(M))$ by $\mathscr{C}^{\#}(M)$. Let $R(M)$ be the quotient of $\mathscr{C}_{0}^{\#}(M)$ by the 2-sided ideal generated by the elements $\bar{K}_{e} \bar{K}_{w}$, where $e$ is the neutral element in $\pi_{1}(M)$ and $w \in$ $\pi_{1}(M)$ is arbitrary. For nonorientable $M$, we have $R(M)=\mathscr{C}_{0}^{\#}(M)$ because a link $L$ one of whose components is a trivial knot, satisfies $2 L=0 \in \mathscr{C}(M)$ by the relation (Bas) and isotopies.

We choose $\hat{\mathscr{Y}}$ as in Section 3. Recall the definition of $\theta$ from page 5 . With the notation above we can state the following theorem.

THEOREM 4.1. Let $\Sigma=P^{2}$ or let $\Sigma$ be a compact connected surface with boundary. Then there exists a unique $\mathbb{Z}[z]$-linear map,

$$
\nabla: \mathscr{C}(\Sigma \times I) \longrightarrow \mathbb{Z}[z] \otimes_{\mathbb{Z}} R\left(\sum \times I\right),
$$

satisfying $\nabla(L)=1 \otimes \bar{L}$ for all $\hat{\mathscr{S}}$-descending links $L$ with $\theta(\bar{L}) \neq 0$. The map $\nabla$ induces an isomorphism $\mathfrak{C}^{\#}(\Sigma \times I) \cong \mathbb{Z}[z] \otimes_{\mathbb{Z}} R\left(\sum \times I\right)$.

Theorem 4.1 is proven in Section 8. In Sections 5 and 6, we prove the uniqueness of $\nabla$. This part of the proof contains a constructive algorithm for the computation of $\nabla(L)$. In Sections 7 and 8, we prove the existence of $\nabla$. For this part of the proof, we use a universal Vassiliev invariant $Z_{\Sigma \times I}$.

As a first example, observe that for $\Sigma=I^{2}$ we have $\nabla(L)=\tilde{\nabla}(L) \bar{K}_{e}$ where $\tilde{\nabla}(L)$ is the usual Conway polynomial of $L$ (see (1.1)).

For a link $L \subset \Sigma \times I$ we denote the mirror image under reflection in $\Sigma \times\{1 / 2\}$ by $L^{*}$. For a link $L$ with ordered components, we use the same order for the components of $L^{*}$ as for their preimages. For $i=0,1$, denote by $|L|_{i}$ the number of components $K$ of $L$ satisfying $\sigma(K)=i$. Then we have the following theorem about special properties of the Conway skein module for certain surfaces.

THEOREM 4.2. (1) For $\Sigma=I^{2}, S^{1} \times I, P^{2}$, or the Möbius strip $X$, the map $\nabla$ satisfies $\nabla(L)=1 \otimes \bar{L}$ and $\nabla\left(L_{1} L_{2}\right)=\nabla\left(L_{1}\right) \nabla\left(L_{2}\right)$ for all $\hat{\mathscr{Y}}$-descending links $L, L_{1}, L_{2}$.

(2) For $\Sigma=P^{2}$ or $\Sigma=X, L=(-1)^{|L|_{0}} L^{*} \in \mathscr{C}^{\#}\left(\sum \times I\right)$.

Theorem 4.2 is proven in Section 8. Notice that we do not require that $\theta(L) \neq 0$ in part (1) of the theorem. In the case where $\Sigma=I^{2}$ we have the well known symmetry property $L^{*}=(-1)^{|L|+1} L$ similar but different from the formula in part (2) of Theorem 4.2, whereas for other surfaces no formula of this type is valid. 
Let $L$ be an ordered based link in $P^{2} \times I$ represented by a link in $X \times I$ as in Figure 4.1, where $X=P^{2} \backslash D^{2}$ is the Möbius strip. Let $L^{\prime}$ be a link in $I^{2} \times I$ as in Figure 4.1, where the parts of the two diagrams consisting of a box labeled $T$ are identical.
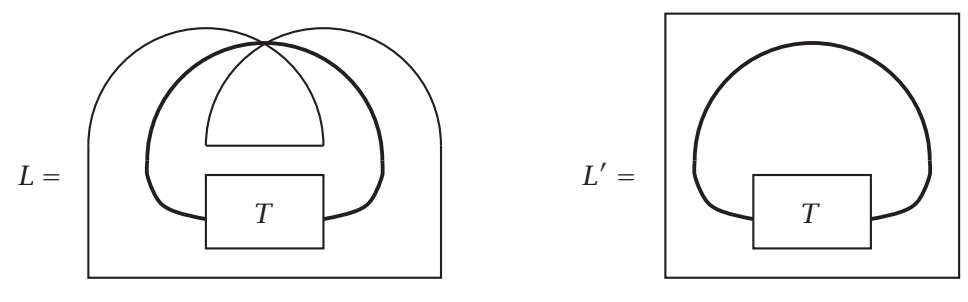

FIGURE 4.1. A link in $P^{2} \times I$ and a link in $I^{2} \times I$.

If $L$ is a knot, then we have $\nabla(L)=\tilde{\nabla}\left(L^{\prime}\right) \bar{K}_{s}$, where $s$ denotes the unique nontrivial element of $\pi_{1}\left(P^{2}, *\right)$. In general, we can compose $\nabla$ with the invariant $C(L)$ (see (2.7)) and obtain

$$
\nabla(C(L))= \begin{cases}\left\{\tilde{\nabla}\left(L^{\prime}\right) \bar{K}_{s}\right\}, & \text { if } L \text { is a knot } \\ \left\{ \pm \tilde{\nabla}\left(L^{\prime}\right) \bar{K}_{s}\right\}, & \text { otherwise. }\end{cases}
$$

The example above is related to a simple special case of the following theorem.

THEOREM 4.3. Let $K$ be a knot in $P^{2} \times I$ with $\nabla(K)=f(z) \bar{K}_{s}$. Then

$$
\nabla\left(p^{-1}(K)\right)=f(z)^{2} \bar{K}_{e}
$$

where $p: S^{2} \times I \rightarrow P^{2} \times I$ denotes the 2-fold covering map.

Theorem 4.3 is proven in Section 9 using relations between Vassiliev invariants of $K$ and $p^{-1}(K)$.

Recall that a knot $K$ in $S^{3}$ is called strongly amphicheiral, if there exists an orientation-reversing involution $i$ of $S^{3}$ with $i(K)=K$. We say that $K$ is strongly positive amphicheiral, if there exists an involution $i$ as above that preserves the orientation of $K$. We have the following corollary.

COROLLARY 4.4. Let $K$ be a strongly positive amphicheiral knot in $S^{3}$. Then $\tilde{\nabla}(K)=$ $f(z)^{2}$ for some $f \in \mathbb{Z}[z]$.

Proof. Strongly positive amphicheiral knots in $S^{3}$ are in one-to-one correspondence with knots $K$ in $P^{2} \times I$ with $\sigma(K)=1$. To see this, recall that the set of fixed points of an orientation-reversing involution $i$ of $S^{3}$ is either $S^{0}$ or $S^{2}$ (see [21]). For strongly positive amphicheiral knots $K$, it is sufficient to consider involutions $i$ with fixed point set $S^{0} \subset S^{3}$ and $K \cap S^{0}=\varnothing$. By a theorem of Livesay (see [17, 20]), we then have $\left.\left(S^{3} \backslash S^{0}\right) / i \cong P^{2} \times\right] 0,1$ [. The corollary follows by applying Theorem 4.3 to the image of $K$ in $\left(S^{3} \backslash S^{0}\right) / i$.

Corollary 4.4 was proven directly by Kawauchi and Hartley using the Blanchfield pairing (see $[6,9,12]$ ). Theorem 4.3 contains the stronger statement that $f(z)$ itself 
can be calculated using a skein relation. It would be interesting to have a direct skeintheoretical proof of Theorem 4.3.

5. Generators of $\mathscr{C}(\Sigma \times I)$. In general, we can show that all sets of knots $\mathscr{K}$, for which $\{\alpha(K) \mid K \in \mathscr{K}\}$ contains a set of representatives of conjugacy classes of $\pi_{1}(\Sigma)$, generate a dense subalgebra of the $z$-adic completion $\lim \mathfrak{b}(\Sigma \times I) / z^{n} \mathscr{C}(\Sigma \times I)$ of $\mathscr{C}(\Sigma \times I)$. As we will see, by the following example, the set $\mathscr{K}$ does not necessarily generate the $\mathbb{Z}[z]$-module $\mathscr{C}\left(\sum \times I\right)$. Choose a generator $s \in \pi_{1}\left(S^{1} \times I, *\right)$. Denote the descending knot in $S^{1} \times I^{2}$ belonging to $s^{n}$ by $K_{n}^{\prime}$. There are knots $K_{n}^{\prime \prime}$ in $S^{1} \times I^{2}$ that are a connected sum of $K_{n}^{\prime}$ and a Whitehead knot in $S^{1} \times I^{2}$ such that the equation $K_{n}^{\prime \prime}=K_{n}^{\prime}-$ $z K_{\operatorname{sgn}(n)(|n|+1)}^{\prime} K_{-\operatorname{sgn}(n)}^{\prime}$ holds in $\mathscr{C}\left(S^{1} \times I^{2}\right)$ (see Figure 5.1 for $n=3$ ). Using Theorem 4.1 it is easy to see that the knots $K_{n}^{\prime \prime}(n \in \mathbb{Z})$ do not generate $\mathscr{C}\left(S^{1} \times I^{2}\right)$ as an algebra, but $\left\{\alpha\left(K_{n}^{\prime \prime}\right) \mid n \in \mathbb{Z}\right\}=\pi_{1}\left(S^{1} \times I\right)$.

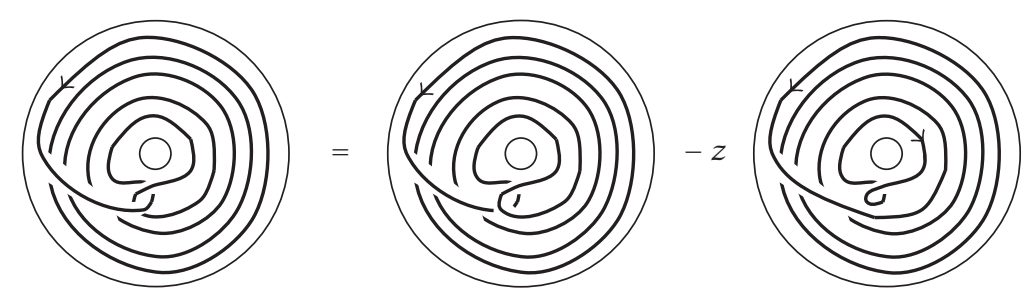

FIGURE 5.1. $K_{3}^{\prime \prime}=K_{3}^{\prime}-z K_{4}^{\prime} K_{-1}^{\prime} \in \mathscr{C}(\Sigma \times I)$.

The knots $K_{n}^{\prime \prime}$ from the previous example are not descending. For a set $\hat{\mathscr{Y}}^{\prime}$ consisting of cyclically reduced representatives of conjugacy classes in $\pi_{1}(\Sigma)$, where $\Sigma$ is a decomposed surface, we can show using Theorem 4.1 that the descending knots $K_{w}$ $\left(w \in \hat{\mathscr{S}}^{\prime}\right)$ generate $\mathbb{Q}(z) \otimes_{\mathbb{Z}[z]} \mathscr{C}(\Sigma \times I)$ as a $\mathbb{Q}(z)$-algebra, where $\mathbb{Q}(z)$ is the quotient field of $\mathbb{Z}[z]$. The knots $K_{w}\left(w \in \hat{\mathscr{Y}}^{\prime}\right)$ do not necessarily generate $\mathscr{C}(\Sigma \times I)$ as a $\mathbb{Z}[z]$ algebra. For example, let $\Sigma$ be a disc with three holes decomposed such that $s\left(x_{i}\right)=i$, $d\left(x_{i}\right)=7-i(i=1,2,3)$. By Theorem 4.1 and Proposition 2.2, there exists a basis of the $\mathbb{Z}[z]$-module $\mathscr{C}^{\#}(\Sigma \times I)$ containing the element $K_{x_{1} x_{3}^{-1} x_{2}}^{*}-K_{x_{1} x_{2} x_{3}^{-1}}^{*}$, where $K^{*}$ denotes the mirror image of $K$ with respect to $\Sigma \times\{1 / 2\}$. Let $\left\{x_{2} x_{1} x_{3}^{-1}, x_{3}^{-1} x_{1} x_{2}\right\} \subset \hat{\mathscr{Y}}^{\prime}$. If the knots $K_{w}\left(w \in \hat{\mathscr{S}}^{\prime}\right)$ would generate $\mathscr{C}(\Sigma \times I)$ we see, by Theorem 4.1 , that we could find a basis of $\mathscr{C}^{\#}(\Sigma \times I)$ containing the element $K_{x_{2} x_{1} x_{3}^{-1}}-K_{x_{3}^{-1} x_{1} x_{2}}$. But this is impossible because we can show by a computation that

$$
K_{x_{2} x_{1} x_{3}^{-1}}-K_{x_{3}^{-1} x_{1} x_{2}}=\left(1+z^{2}\right)\left(K_{x_{1} x_{3}^{-1} x_{2}}^{*}-K_{x_{1} x_{2} x_{3}^{-1}}^{*}\right) \in \mathscr{C}\left(\sum \times I\right) .
$$

According to the following lemma the set $\hat{\mathscr{S}}$ chosen in Section 3 has better properties.

LEMMA 5.1. The descending knots $K_{w}(w \in \hat{\mathscr{S}})$ generate $\mathscr{C}(\Sigma \times I)$ as a $\mathbb{Z}[z]$-algebra.

Proof. Let $L$ be a diagram of an ordered based link. For the proof we use the following strategy: we never increase the number of crossings of $L$, and make computations modulo diagrams with less crossings. This allows us to make crossing changes. 
We prove the lemma by induction, where the induction base is given by the following arguments for link diagrams without crossings, and the induction step is given by the same arguments for link diagrams with crossings.
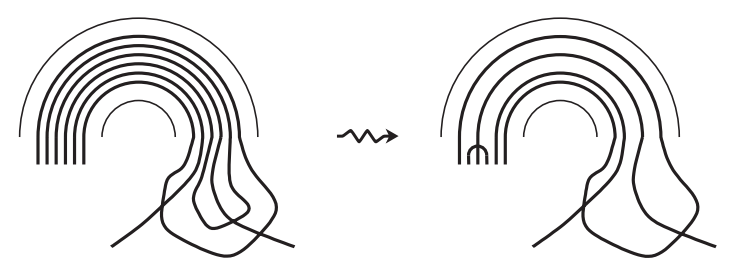

FIGURE 5.2. Eliminating cancellations of generators of $\pi_{1}(\Sigma)$.

CASE $1 \partial \Sigma \neq \varnothing$.

STEP 1. We apply an isotopy that does not increase the number of double points in $p_{\Sigma}(L)$ such that the projection of $L$ to $\bigcup_{i=1}^{k} B_{i}$ consists of parallel strands connecting points in $I_{S\left(x_{i}\right)}$ with points in $I_{d\left(x_{i}\right)}$. Then we pass from $L$ to a diagram of a product of knots by crossing changes.

STEP 2. Each component of $L$ represents a unique word in the generators $x_{i}$ of $\pi_{1}(\Sigma)$. We claim that we can replace each of these components by a diagram of a based knot $K$ representing a cyclically reduced word in $\pi_{1}(\Sigma)$ : for words that are not cyclically reduced, we find a segment $h$ of $K$ corresponding to a cancellation such that $p_{\Sigma}(h)$ connects two points $P_{1}, P_{2} \in I_{v}$ for some $v$, and all the parts of $p_{\Sigma}(K)$ that go into $B_{0}$ between $P_{1}$ and $P_{2}$ intersect $p_{\Sigma}(h)$ (see Figure 5.2).

The part $h$ can first be moved to a suitable height by making crossing changes and then be pulled back by applying an isotopy that does not increase the number of crossings as shown in Figure 5.2. This will remove one cancellation from the word in the generators $x_{i}, x_{i}^{-1}$ corresponding to the knot projection. We continue by induction on the number of cancellations to pass to a cyclically reduced word.

STEP 3. Recall the definitions of $\dot{w}$ and $\hat{w}$ for $w \in \pi_{1}(\Sigma)$ from (3.4). We continue with the modification of each based knot $K$ in $L$ by moving the basepoint of $K$ until $w=\hat{w}$ where $w=\alpha(K)$. By relation (Bas), this only contributes a factor \pm 1 . We can further achieve that the projection $A$ of the basepoint of $K$ to $\Sigma$ lies in $I_{S(\dot{w})}$. Assume that there are points in $p_{\Sigma}(K) \cap I_{S(\dot{w})}$ to the left of $A$ in our pictures of $\Sigma$. Let $P$ be the left neighbor of $A$ in $p_{\Sigma}(K) \cap I_{\mathcal{S}(\dot{w})}$. Notice that there may be points of $p_{\Sigma}(L \backslash K) \cap I_{\mathcal{S}(\dot{w})}$ between $P$ and $A$. Let $v$ be the element of $\pi_{1}(\Sigma)$ that is represented by $p_{\Sigma}(K)$ with basepoint $P$ and with an orientation such that at $P$ the curve $p_{\Sigma}(K)$ enters the band. By the definition of the representative $\hat{w}$ we have $v \geq \dot{w}$. Assume first that $v>\dot{w}$. We have $v(1)=\dot{w}(1)$. Therefore, following the two strands of $p_{\Sigma}(K)$ starting by entering a band at $P$ and $A$, we follow paths $p$ and $a$, respectively, that pass through the same bands until we find a crossing $c$ between them. Denote that part of $L$ by $T$ whose projection lies in a small neighborhood of the triangle bounded by $a, p$, and $I_{S}(\dot{w})$ with corners $P, A$, and $c$. By crossing changes we pass to a suitable height on $T$ and then pull back this part by an isotopy as shown in Figure 5.3 until the points $P$ and $A$ are interchanged. The projection of $T$ arrives very close to $I_{S(\dot{w})}$ in $B_{0}$ such that no crossings with the remaining part of $p_{\Sigma}(L)$ can appear. 


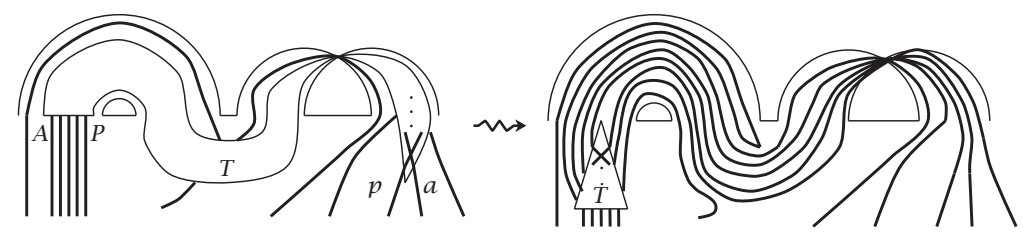

FIGURE 5.3. Moving the basepoint to the left in $p_{\Sigma}(K) \cap I_{S(\dot{w})}$.

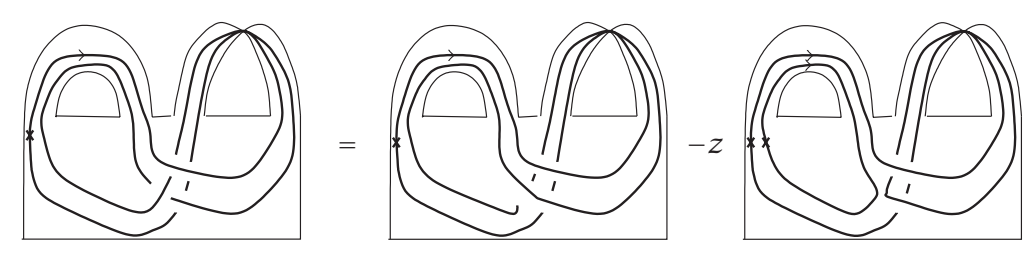

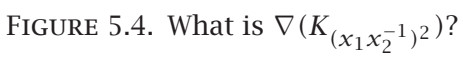

If $v=\dot{w}$, then we push the basepoint along $K$ until its projection arrives at $P$. Therefore, in any case this modification does not increase the number of crossings of $K$. This implies that by induction, we can assume that there are no points in $p_{\Sigma}(K) \cap$ $I_{S(\dot{w})}$ to the left of $A$. Then we pass from $K$ to a descending knot by crossing changes. Since this works for all the components of $L$ we have proven the lemma in the case where $\partial \Sigma \neq \varnothing$.

CASE $2 \Sigma=P^{2}$. We choose an open disc $D \subset P^{2}$. Then $P^{2} \backslash D=X$ is the Möbius strip. It is easy to see that every link in $P^{2} \times I$ can be represented by a link in $X \times I$ and that a descending link in $X \times I$ is isotopic to a descending link in $P^{2} \times I$. Therefore, we conclude using this lemma for $\Sigma=X$.

Let $\Sigma$ be a surface with nonempty boundary. Let $w \in \pi_{1}(\Sigma) \backslash\{e\}$. In the free group $\pi_{1}(\Sigma)$, the normalizer $N(w)$ is isomorphic to $\mathbb{Z}$. A generator $z$ of $N(w)$ is characterized by the property that $w=z^{k}$ where $k \in \mathbb{Z}$ and $|k|$ is as large as possible. If $\sigma(z)=1$ and $\sigma(w)=0$, then by Proposition 2.4 we have $\bar{K}_{w}=0 \in \mathscr{C}_{0}^{\#}\left(\sum \times I\right)$. Nevertheless, we may have $K_{w} \neq 0 \in \mathscr{C}^{\#}\left(\sum \times I\right)$. Hence it is not obvious how to calculate $\nabla\left(K_{w}\right)$ using only the properties of $\nabla$ given in Theorem 4.1. Our next goal will be to see why the equation in Figure 5.4 implies that $\nabla\left(K_{\left(x_{1} x_{2}^{-1}\right)^{2}}\right)=-z \bar{K}_{x_{1} x_{2}^{-2}} \bar{K}_{x_{1}}$. In the rest of this section we will reduce this question to a problem in $\mathscr{C}^{\#}(X \times I)$, where $X$ is the Möbius strip.

Fix a generator $s$ of $\pi_{1}(X)$ and denote the descending knot $K_{s} n$ by $K_{n}$. For $v \in \pi_{1}(\Sigma)$ with $\sigma(v)=1$ we have an embedding $i_{v}: X \times I \rightarrow \Sigma \times I$ such that $i_{v}\left(K_{1}\right)=K_{v}$. Up to isotopy, $i_{v}$ is uniquely determined by $v$ (since $\sigma(v)=1$ no framing is needed). By Proposition 2.2 the embedding $i_{v}$ induces a map between skein-modules $i_{v *}: \mathscr{C}(X \times$ $I) \rightarrow \mathscr{C}(\Sigma \times I)$. We can assume that $i_{v *}$ is $\mathbb{Z}[z]$-linear by making a suitable choice of the oriented subset $U$ of $X \times I$. For $w \in \pi_{1}(\Sigma) \backslash\{e\}$, there exists a unique generator $u$ of $N(w)$ such that $w=u^{n}$ for $n \in \mathbb{N}$. If $\sigma(u)=1$ and $\sigma(w)=0$, then we define 
the cabled descending knot as $\hat{K}_{w}=\left(i_{u}\right)_{*}\left(K_{n}\right)$. We define $\hat{K}_{w}=K_{w}$ in the remaining cases. Using this notation we can rewrite the equation in Figure 5.4 as

$$
K_{\left(x_{1} x_{2}^{-1}\right)^{2}}=\hat{K}_{\left(x_{1} x_{2}^{-1}\right)^{2}}-z K_{x_{1} x_{2}^{-2}} K_{x_{1}}=\hat{K}_{\left(x_{1} x_{2}^{-1}\right)^{2}}-z \hat{K}_{x_{1} x_{2}^{-2}} \hat{K}_{x_{1}} .
$$

LEMMA 5.2. The knots $\hat{K}_{w}(w \in \hat{9})$ generate $\mathscr{C}\left(\sum \times I\right)$ as a $\mathbb{Z}[z]$-algebra.

Proof. For $\Sigma=P^{2}$ there is nothing to prove. Assume that $\partial \Sigma \neq \varnothing$. Let $L$ be a link in $\Sigma \times I$. By the first two steps of the proof of Lemma 5.1 we can assume that the connected components of $L$ represent cyclically reduced words in $\pi_{1}(\Sigma)$. Applying the following Steps 1 and 2 to all the components $K$ of $L$ will imply the lemma by induction on the number of crossings:

STEP 1 (collect all starting points on the left). Choose a maximal $k>0$ such that $\dot{w}=$ $\left(a_{1} \cdots a_{n}\right)^{k} \in \pi_{1}(\Sigma)$ where $w=\alpha(K)$ and $a_{i} \in\left\{x_{j}^{ \pm 1}\right\}$. Assume that $\sigma\left(a_{1} \cdots a_{n}\right)=1$ (otherwise there is nothing to do). Consider the $k$ points $A_{i}$ on $K$ such that $p_{\Sigma}\left(A_{i}\right) \in$ $I_{s\left(a_{1}\right)}$ and $p_{\Sigma}(K)$ with each of these points represents $\hat{w}$. We want to move all the points $p_{\Sigma}\left(A_{i}\right)$ to the left side in $p_{\Sigma}(K) \cap I_{S\left(a_{1}\right)}$ without increasing the number of crossings of $L$ : if this is not already the case, then choose $j$ such that the point $P$ to the left of $A:=p_{\Sigma}\left(A_{j}\right)$ in $p_{\Sigma}(K) \cap I_{s\left(a_{1}\right)}$ is not one of the points $p_{\Sigma}\left(A_{i}\right)$. Let $\left(b_{1} \cdots b_{n}\right)^{k}$ be the element of $\pi_{1}(\Sigma)$ represented by $p_{\Sigma}(K)$ with respect to $P$ and oriented such that it leaves $B_{0}$ at $P$. Since we use this orientation of $p_{\Sigma}(K)$, the word $b_{1} \cdots b_{n}$ is obtained from $a_{1} \cdots a_{n}$ by a cyclic permutation.

The minimality of $\dot{w}$ and the maximality of $k$ imply that $a_{1} \cdots a_{n}<b_{1} \cdots b_{n}$. We have $b_{1}=a_{1}$. Therefore, we can follow $p_{\Sigma}(K)$ on two strands along $b_{1} \cdots b_{m}=$ $a_{1} \cdots a_{m}(m<n)$ starting at $P$ and $A$ until we find a crossing $c$ between these two strands. As in Step 3 of the proof of Lemma 5.1 we make crossing changes and then pull back this crossing and possibly some other parts of $L \backslash K$ by an isotopy until the points $P$ and $A$ are interchanged (see Figure 5.3). With this modification we do not increase the number of crossings of $L$ and the point $A$ has moved one step to the left in $p_{\Sigma}(K) \cap I_{S\left(a_{1}\right)}$. We now verify that no other point $A^{\prime}=p_{\Sigma}\left(A_{j^{\prime}}\right)$ has moved to the right during this operation: assume that we reach $A^{\prime}$ after starting at $P$ and traveling along $b_{1} \cdots b_{m^{\prime}}$ with $0<m^{\prime} \leq m$. Then for $x=a_{1} \cdots a_{m^{\prime}}$ we have either $a_{1} \cdots a_{n}=x y x$ or $a_{1} \cdots a_{n}=x y x^{-1}$. We can exclude the second case because this would imply that $x=a_{1} \cdots a_{m^{\prime}}=b_{1} \cdots b_{m^{\prime}}=x^{-1}$, which is impossible. By the minimality of $\dot{w}=(x y x)^{k}$ and the maximality of $k$ we have $x y x<y x x$ and $x y x<x x y$. From $x y x<y x x$, we deduce that $x y<y x$. Then $x y x<x x y$ and $y x>x y$ implies that $\sigma(x)=1$. Therefore, starting at $P$ and $A$ and traveling along $x$, the first strand reaches $I_{S\left(a_{1}\right)}$ to the right of the second one. This means that by pulling back the crossing $c$ we move the two points $A$ and $A^{\prime}$ to the left (see Figure 5.5 for an example). We can continue by induction until all points $p_{\Sigma}\left(A_{i}\right)$ are on the left side of $p_{\Sigma}(K) \cap I_{S}\left(a_{1}\right)$.

STEP 2 (move component into cabling position). Successively, for $i=2, \ldots, n$, we consider all parts $s_{v}$ of $K$ such that the projections $p_{\Sigma}\left(s_{v}\right)$ connects some interval $I_{j}$ with $I_{s\left(a_{i}\right)}$ inside of $B_{0}$. By crossing changes we move all the $s_{v}$ to a suitable height and then push the part $T$ containing all crossings between these strands across the band at $I_{S\left(a_{i}\right)}$. This isotopy can be chosen in the following way such that the total number of crossings of $L$ does not increase: after being pushed across the band the 

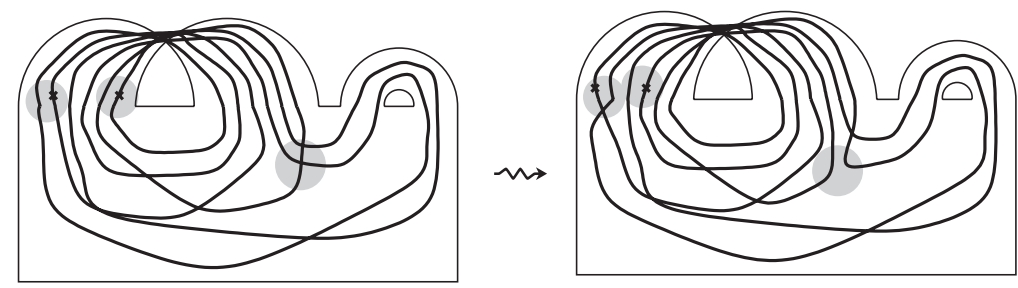

FIGURE 5.5. Applying Step 1 to the leftmost point of $p_{\Sigma}(K) \cap I_{1}$.

part $T$ arrives very close to $I_{d\left(a_{i}\right)} \times I$ and the strands that now enter into $I_{S\left(a_{i}\right)}$ without intersections in their projection to $\Sigma$ can be moved into a position such that they coincide with the original parts of $T$ except in the neighborhoods of crossings and except for orientations.

After having done the previous modification for $i=2, \ldots, n$ we make crossing changes such that $K=i_{a_{1} \cdots a_{n *}}\left(K^{\prime}\right)$, where $K^{\prime}$ is a knot in $X \times I$. Applying the arguments in the proof of Lemma 5.1 for $\Sigma=X$ to $K^{\prime}$ we see that $K$ is equal to $i_{a_{1} \cdots a_{n *}}\left(K_{k}\right)=$ $\hat{K}_{w}$ modulo diagrams with less crossings.

In a diagram of a product of knots we can permute two components modulo diagrams with less crossings. By the relation (Ord) this allows to express $2 K_{w}^{2}(\sigma(w)=1)$ by diagrams with less crossings. This can be used in the calculation of $\nabla(L)$ by working with $\mathbb{Z}[1 / 2][z] \otimes R\left(\sum \times I\right)$. In order to prove that $\nabla$ takes values in $\mathbb{Z}[z] \otimes R\left(\sum \times I\right)$ we have to be more careful. Since the presentation of $\mathscr{C}_{0}(\Sigma \times I)$ by generators and relations (see the proof of Proposition 2.4) shows that $K_{w}^{2} \not \equiv 0 \bmod z$ we pass to a quotient of $\mathscr{C}(\Sigma \times I)$. Let $J$ be the 2-sided ideal of $\mathscr{C}(\Sigma \times I)$ generated by elements $i_{*}\left(K_{1}^{2}\right)$ for all embeddings $i: X \times I \rightarrow \Sigma \times I$ as in Proposition 2.2. Then we have the following lemma.

LEMMA 5.3. The $\mathbb{Z}[z]$-module $\mathscr{C}\left(\sum \times I\right) / J$ is generated by the following set of links:

$$
\left\{\hat{K}_{w_{1}} \cdots \hat{K}_{w_{n}} \mid n \in \mathbb{N}, w_{i} \in \hat{\mathscr{Y}}, w_{i} \leq w_{i+1},\left(w_{i}=w_{i+1} \Longrightarrow \sigma\left(w_{i}\right)=0\right)\right\}
$$

Proof. The proof of Lemma 5.2 shows that the $\mathbb{Z}[z]$-module $\mathscr{C}\left(\sum \times I\right)$ is generated by the links $\hat{K}_{w_{1}} \cdots \hat{K}_{w_{n}}$ with $n \in \mathbb{N}, w_{i} \in \hat{\mathscr{Y}}$, and $w_{i} \leq w_{i+1}$. Consider the links $L$ of the form $L^{\prime} K^{\prime} K^{\prime \prime} L^{\prime \prime}$ where $L^{\prime}$ and $L^{\prime \prime}$ are ordered based links and $K^{\prime}=K^{\prime \prime}=\hat{K}_{w}$ with $\sigma(w)=1$. For $\sigma(w)=1$ we always have $K_{w}=\hat{K}_{w}$. As shown in the proof of Lemma 5.1 we may assume that in a diagram of $L$ the projections of the components $K^{\prime}$ and $K^{\prime \prime}$ of $L$ represent cyclically reduced words in $\pi_{1}(\Sigma)$. Furthermore, we may assume that the projections $A^{\prime}$ and $A^{\prime \prime}$ of the basepoints of $K^{\prime}$ and $K^{\prime \prime}$ lie in $I_{S(w)}$. When we follow the two strands of $p_{\Sigma}\left(K^{\prime} \cup K^{\prime \prime}\right)$ starting by entering a band at $A^{\prime}$ and $A^{\prime \prime}$, we follow the paths $a^{\prime}$ and $a^{\prime \prime}$, respectively, that pass through the same bands until we find a crossing $c$ between them. We pull this crossing back as shown in Figure 5.3. We continue with the modification of $L$ as in Step 2 of the proof of Lemma 5.2. We obtain that $L$ is equal to $L^{\prime} i_{*}\left(K_{1}^{2}\right) L^{\prime \prime}$ modulo diagrams with less crossings, where we have $\alpha\left(i_{*}\left(K_{1}\right)\right)=w$ and where we can choose the embedding $i: X \times I \rightarrow \Sigma \times I$ such that $i_{*}\left(K_{1}\right)$ is a knot descending with respect to its basepoint (but in general not a 
descending knot). The link $L^{\prime} i_{*}\left(K_{1}^{2}\right) L^{\prime \prime}$ lies in the ideal $J$. We obtain the lemma by induction on the number of crossings.

6. Generators of $\mathscr{C}^{\#}(X \times I)$. We show by making computations in $\mathscr{C}^{\#}(X \times I)$ that the knots $\hat{K}_{w}$ with $\hat{K}_{w} \neq K_{w}$ are 0 in $\mathscr{C}^{\#}(\Sigma \times I)$. Verifying directly that

$$
\left(z^{2}+4\right) i_{*}\left(K_{1}^{2}\right)=0 \in \mathscr{C}(\Sigma \times I)
$$

gives a good impression of the ideas used in this section. Readers that are mainly interested in the case $\Sigma=P^{2}$ can concentrate on the verification of (6.1).

Recall that we denote the descending knot $K_{s^{n}}$ for a fixed choice of a generator $s \in \pi_{1}(X)$ by $K_{n}$. In this section, we represent ordered based links in $X \times I$ by drawing only a part of them as shown in Figure 6.1. We say that we represent an ordered based link in $L$ as the closure of a tangle.

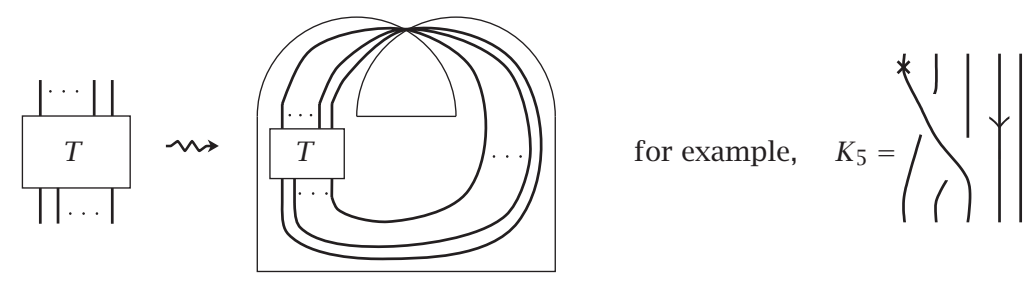

FiguRE 6.1. The closure of a tangle.

The tangle $T$ must have the same number $n$ of endpoints at the top and at the bottom and the strand at the $i$ th endpoint at the top (always by counting the endpoints from the left to the right side) is directed downwards if and only if the $(n+1-i)$ th strand at the bottom is directed downwards $(i=1, \ldots, n)$. The ordered set of basepoints of $T$ is in bijection with the components of the closure of $T$.

The following lemmas are important for determining $\mathscr{C}^{\#}(X \times I)$. They also provide shortcuts in the computation of the Conway polynomial.

LEMMA 6.1. For all $k, n, m \in \mathbb{Z}$ the following identities hold in $\mathfrak{}^{\#}(X \times I)$ :

$$
K_{2 k}=0, \quad K_{2 n+1} K_{2 m+1}=-K_{2 m+1} K_{2 n+1} .
$$

As a preparation for the proof of Lemma 6.1 we first prove the following lemma.

LEMMA 6.2. Assume that $K_{2 n}=0 \in \mathscr{C}^{\#}(X \times I)$ for all $n$ with $-k<n<k$. Then for $i \in \mathbb{Z}$ with $-k<i<k$ the following identities hold in $\mathscr{C}^{\#}(X \times I)$ :

(1) if a link $L$ is a closure of a tangle $T$ on $2 i$ strands as in Figure 6.2 with $r=i$, then $L=0$;

(2) if a knot $K$ is a closure of a tangle on $l \in\{2 i+1,2 i+2\}$ strands as in Figure 6.2 with $r=i+1, K$ is descending with respect to its basepoint, the basepoint lies on one of the first $i+1$ strands of $K$, and $K$ is homotopic to $K_{m}$, then $K=K_{m}=K_{m}^{*}$.

Proof. (1) Since $2 i$ is even and the diagram of $L$ is as shown in Figure 6.2, we can pass from $L$ to a product $\pm K_{2 i_{1}} \cdots K_{2 i_{r}}$ with $-i<i_{v}<i$ by crossing changes and by 

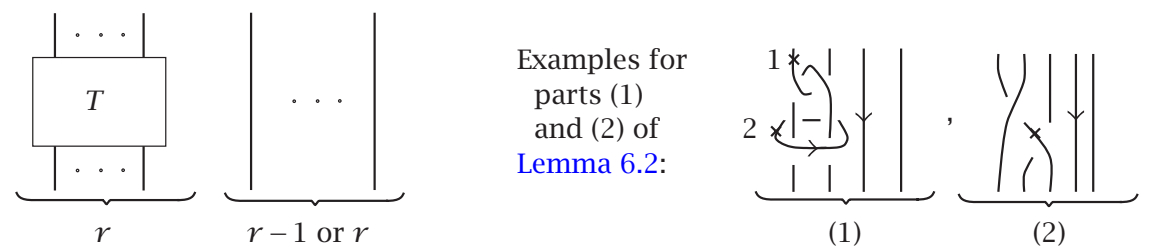

(1)

(2)

FIGURE 6.2. Special tangles on an even or odd number of strands.

the relation (Bas). If a crossing of $L$ is spliced, then we obtain again a closure of a tangle as in Figure 6.2. By induction on the number of crossings of $L$, we can expand $L$ as a linear combination over $\mathbb{Z}[z]$ of monomials $K_{2 j_{1}} \cdots K_{2 j_{m}}$ with $-i<j_{i}<i$. Under the hypotheses of Lemma 6.2 all these monomials equal 0 in $\mathscr{C}^{\#}(X \times I)$.

(2) Let $K$ be as in part (2) of Lemma 6.2. When we push the basepoint of $K$ along the generator $s$ of $\pi_{1}(X)$ and $l$ is odd, then the relation (Bas) contributes the sign $(-1)^{1 \cdot(l-1)}=1$. If $l$ is even, then we will see in the following that we will push the basepoint along $s$ an even number of times, because the basepoint is on one of the first $i+1$ strands of $K$. This will not give a sign contribution either. If the basepoint passes through a crossing, then by changing this crossing, the knot will become descending with respect to the new basepoint. If the crossing is spliced, then we obtain a product of two knots that are descending with respect to their basepoints. One of these knots is isotopic to $K_{0}=0$ or satisfies the conditions from part (1) of the lemma. Therefore, we do not change $K$ if we push the basepoint along $K$ and change crossings until the basepoint is close to the left boundary of $X$ in our pictures. This implies that $K=K_{m}$ if $K$ is homotopic to $K_{m}$ (the number $m$ satisfies $-l \leq m \leq l$ and $l \equiv m \bmod 2$ ). There exists a diagram of $K_{m}$ as a closure of a tangle as in Figure 6.2 on $m$ strands having $[(m-1) / 2]$ crossings such that by changing arbitrary crossings and by splicing one arbitrary crossing we obtain a product of two knots (see Figure 6.1 for $m=5$ ). Using this we obtain $K_{m}=K_{m}^{*}$ by similar arguments as above.

Proof of Lemma 6.1. We prove the lemma by induction. We have $K_{0}=0$. Assume that the lemma is true for all $k, n, m$ with $|k| \leq \ell$ and $|n|+|m|<\ell$. We have to prove the lemma for $|k|-1=\ell=|n|+|m|$. Since the inversion of the orientations of all components of a link induces a $\mathbb{Z}[z]$-linear involution of $\mathscr{C}(M)$, we can assume without loss of generality that $k>0$ and $n \geq 0$. We prove the following two equations:

$$
\begin{gathered}
2 K_{2 k}=-z \sum_{i=0}^{k-1} K_{2 i+1} K_{2(k-i)-1}, \\
K_{2 n+1} K_{2 m+1}+K_{2 m+1} K_{2 n+1}=z p(z) K_{2 k}, \quad \text { for some } p(z) \in \mathbb{Z}[z] .
\end{gathered}
$$

Equations (6.3) and (6.4) imply that $\left(2+z^{2} q(z)\right) K_{2 k}=0$ for some $q(z) \in \mathbb{Z}[z]$. Therefore, the $K_{2 k}=0 \in \mathscr{C}^{\#}(X \times I)$. This, together with (6.4), implies also that $K_{2 n+1} K_{2 m+1}=$ $-K_{2 m+1} K_{2 n+1} \in \mathscr{C}^{\#}(X \times I)$.

We prove (6.3) for $k=3$ in Figure 6.3 and explain afterwards why this equation holds for arbitrary $k$. 

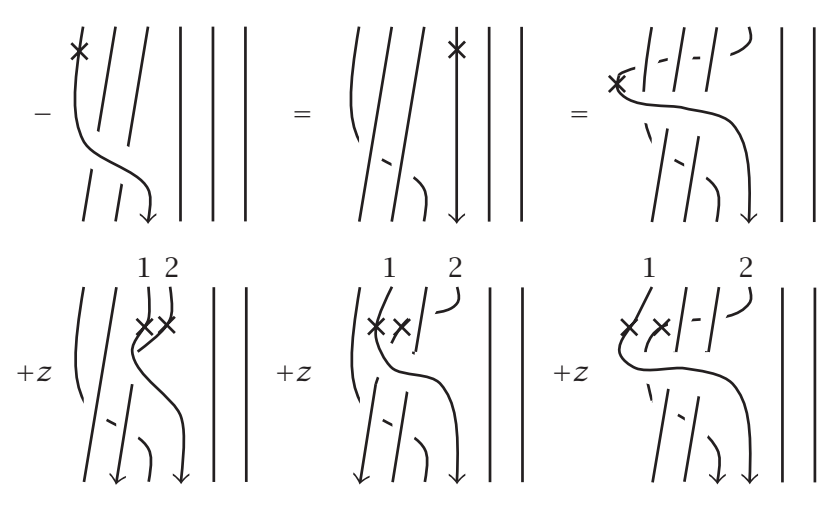

FIGURE 6.3. A refinement of $2 \bar{K}_{2 k}=0 \in \mathscr{C}_{0}(\Sigma \times I)$.

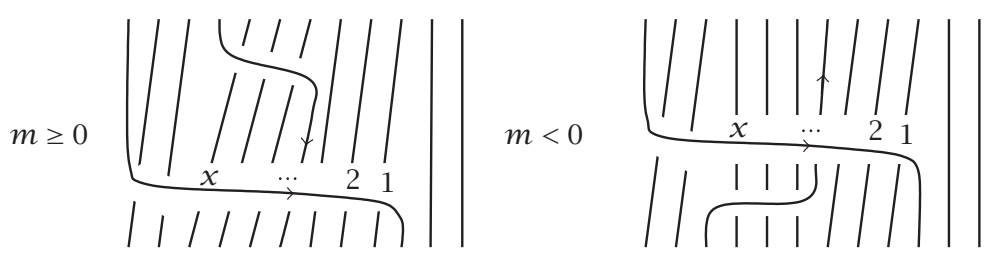

FiguRE 6.4. The links $K_{5} K_{7}$ and $K_{5} K_{-7}$.

The first equality in Figure 6.3 follows in general from part (2) of Lemma 6.2 for $l=2 k$ and from the relation (Bas). The new knot is descending with respect to its basepoint. The second equality follows by applying the skein relation successively $k$ times to crossings that appear when the new basepoint is pulled to the left. When all crossings are changed, then we obtain again $K_{2 k}$. When one of the $k$ crossings is spliced, then we obtain a product of two descending knots each of which satisfies the conditions of part (2) of Lemma 6.2 for odd $l$. This gives us the $k$ product links $K_{i} K_{2 k-i}$ where $i$ runs successively over the numbers $1,3, \ldots, 2 k-1$. Looking at the signs we obtain (6.3).

Proof of (6.4). The link $K_{2 n+1} K_{2 m+1}$ is the closure of a tangle as shown in Figure 6.4. In this diagram the crossings between the two components of $K_{2 n+1} K_{2 m+1}$ are numbered from 1 to $x:=|2 m+1|$. The position of the basepoints on $K_{2 n+1} K_{2 m+1}$ is not important.

Let $D_{i}(n, m)(i=1, \ldots, x=|2 m+1|)$ be the knot obtained by changing the crossings $1,2, \ldots, i-1$ and by splicing the crossing $i$ in Figure 6.4 (see Figure 6.5). For later use, some crossings of the diagram of $D_{i}(n, m)$ are marked by the symbol $*$ in Figure 6.5.

Since we can permute the two components of $K_{2 n+1} K_{2 m+1}$ by changing all crossings between them, we obtain by the skein relation and by relation (Ord) that

$$
K_{2 n+1} K_{2 m+1}=-K_{2 m+1} K_{2 n+1}+z \sum_{i=1}^{|2 m+1|} \epsilon_{i} D_{i}(n, m)
$$




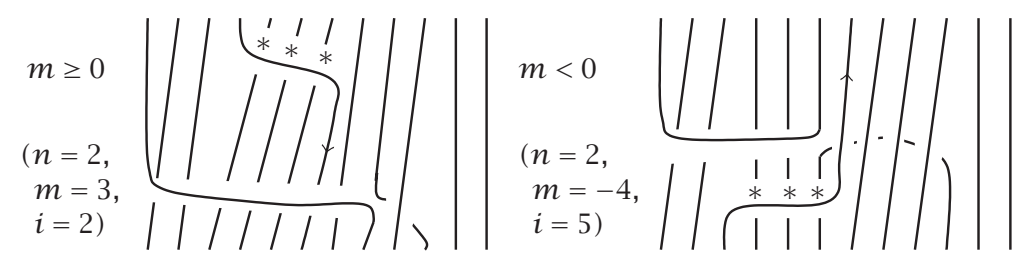

FIGURE 6.5. The diagram $D_{i}(n, m)$.

for some basepoint on $D_{i}(n, m)$ and some signs $\epsilon_{i} \in\{ \pm 1\}$. Equation (6.4) follows from (6.5), the induction hypothesis and the subsequent lemma.

Let $J_{k}$ be the 2 -sided ideal of $\mathscr{C}\left(\sum \times I\right)$ generated by knots $K_{2 n}$ with $-k<n<k$. Recall the definition of the diagram $D_{i}(n, m)$ from the proof of Lemma 6.1 (see Figure 6.5). Then the following holds.

LEMMA 6.3. For $n \in \mathbb{N}, m \in \mathbb{Z}$, and $i \leq|2 m+1|$ then $D_{i}(n, m)=p(z) K_{2 k}+a$, where $p(z) \in \mathbb{Z}[z], k=n+|m|+1$, and $a$ is some element of $J_{k}$.

Proof. A binary tree with root $L=D_{i}(n, m)$ is given by the following recursive description:

(1) we start at the top left of the diagram of $L$ and follow the orientation of the strand until we come back to the point where we started or until we travel along an undercrossing strand of a crossing where we did not travel along the overcrossing strand of that crossing before;

(2a) if we come back to the starting point on $L$ in step 1, then the tree for $L$ consists of a leaf labeled $L$;

(2b) if we reach a crossing first as an undercrossing in step 1, then we apply the relations (Bas) and (Ord) and then a skein relation to this crossing. We obtain $L=$ $\epsilon_{1} L_{1}+\epsilon_{2} z L_{2}$ for some $\epsilon_{j} \in\{ \pm 1\}$. The tree for $L$ consists of a root vertex connected to a tree for $L_{1}$ and to a tree for $L_{2}$.

The knot $D_{i}(n, m)$ is equal to a weighted sum of the labels $L^{\prime}$ of the leaves in this tree with weights $\pm z^{j}$, where $j$ is equal to the number of crossings spliced on the path in the tree leading from the root to $L^{\prime}$. The leaves are labeled links $L^{\prime}$ that are the products $K L^{\prime \prime}$, where $K$ is a descending knot. We further examine the paths in the tree leading from the root to a leaf. There is a unique path in the tree where no crossing is spliced and the label of the leaf at the end of this path is $K_{2 n+2 m+2}$.

If a crossing of $D_{i}(n, m)$ is spliced, then the first one has to be one of the crossings marked by a $*$ in Figure 6.5. Let $D^{s}$ be the resulting link diagram (see Figure 6.6).

For $m \geq 0$ (resp., $m<0$ ) let $B$ be a point on $D^{s}$ near the $(1+n+|m|)$ th endpoint at the bottom (resp., at the top) of $D^{s}$. Traveling along $D^{s}$ as in step 1 of the description of the tree for $D^{s}$, we first pass through a strand of the crossing that was spliced by passing from $K_{2 n+1} K_{2 m+1}$ to $D_{i}(n, m)$. We label a point on this strand by $A$ and a point on the other strand of this spliced crossing by $C$ (see Figure 6.6). When we continue to travel along $D^{s}$, we will arrive first at the point $B$ and then at the point $C$ on $D^{s}$ without the need of a further modification of $D^{s}$. Forgetting the component $K^{\prime}$ of $D^{s}$ containing 


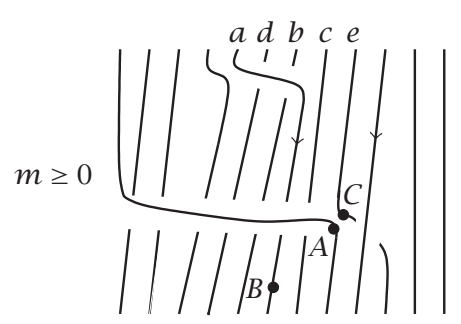

e c $b d a$

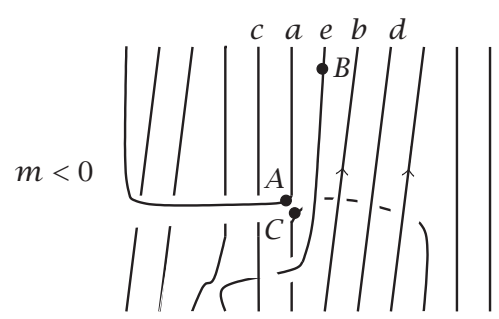

$d \quad b$ e a c

FIGURE 6.6. The diagram $D^{s}$ obtained from $D_{i}(n, m)$.

the points $A, B, C$, we obtain a knot $K^{\prime \prime}$. The upper and lower boundary points of the diagram of $K^{\prime \prime}$ consist of a subset of the corresponding boundary points of $K_{2 m+1}$ in Figure 6.4 , the $\left(\delta_{1 \operatorname{sgn}(m)}+|m|\right)$ th upper and lower boundary points do not belong to $K^{\prime \prime}$ because $B$ lies on $K^{\prime}$, and the strands of $K^{\prime \prime}$ to the right of the point $B$ do not intersect. This implies that $K^{\prime \prime}$ is the closure of a tangle on $2 r$ strands as in Figure 6.2. The link $D^{s}$ is not the product of $K^{\prime}$ and $K^{\prime \prime}$, but the point $C$ lies between the $r$ th and $r+1$ st strand of $K^{\prime \prime}$, and when we travel along $K^{\prime}$ from $C$ back to the starting point we cross $K^{\prime \prime}$ only in strands to the right of $C$. This implies that no matter how $D^{s}$ is modified in the passage from $D^{s}$ to a leaf $L$, the component of $L$ containing the points $A, B, C$ will always be homotopic to $K_{2 j}$ for some $j \in\{n-|m|, \ldots, n+|m|+1\} \subseteq\{-k+1, \ldots, k\}$. We have $K_{2 j} \in J_{k}$ for $|j|<k$. If the label $L$ of the leaf of the tree is of the form $L=K_{2 k} L^{\prime}$ for some link $L^{\prime}$, then $L=K_{2 k}$.

Combining Lemmas 6.1 with 5.3 we obtain the following proposition.

Proposition 6.4. The $\mathbb{Z}[z]$-module $\mathscr{C}^{\#}(\Sigma \times I)$ is generated by the following set of links:

$$
\begin{aligned}
\varphi_{\Sigma}=\left\{K_{w_{1}} \cdots K_{w_{n}} \mid n \in \mathbb{N}, w_{i} \in \hat{\varphi}^{*}, w_{i} \leq w_{i+1},\right. \\
\left.\quad\left(w_{i}=w_{i+1} \Longrightarrow \sigma\left(w_{i}\right)=0\right),\left(w_{i}=e \Longrightarrow i=n=1\right)\right\} .
\end{aligned}
$$

Proof. The cabled descending knots $\hat{K}_{w}$ with $\hat{K}_{w} \neq K_{w}$ are indexed by $w \in \hat{\mathscr{S}} \backslash$ $\left(\hat{\mathscr{S}}^{*} \cup\{e\}\right)$. By definition they are of the form $\hat{K}_{w}=i_{v *}\left(K_{2 n}\right)$ for some $v \in \pi_{1}(\Sigma)$ with $\sigma(v)=1$ and $n>0$. By Lemma 6.1 we have $K_{2 n}=0 \in \mathscr{C}^{\#}(X \times I)$ implying that $\hat{K}_{w}=0 \in \mathscr{C}^{\#}(\Sigma \times I)$. Also by Lemma 6.1 , we have $2 K_{1}^{2}=0 \in \mathscr{C}^{\#}(X \times I)$ implying that $i_{*}\left(K_{1}^{2}\right)=0 \in \mathscr{C}^{\#}(\Sigma \times I)$ for an arbitrary embedding $i: X \times I \rightarrow \Sigma \times I$. This shows that the ideal $J$ of Lemma 5.3 is contained in $\operatorname{Tor}_{\mathbb{Z}[z]}\left(\mathscr{C}\left(\sum \times I\right)\right)$. It is easy to see that for all 3-manifolds $M$ we have $z K_{e} K_{w}=0 \in \mathscr{C}(M)$. For nonorientable $M$ we have $K_{e}=0$ and $e \notin \hat{9}^{*}$. The three arguments from above together with Lemma 5.3 imply the proposition.

7. The weight system of the Conway polynomial. We used ordered based links to define the Conway polynomial $\nabla$ taking values in $\mathbb{Z}[z] \otimes R\left(\sum \times I\right)$. We will construct a map $W_{\mathfrak{g} \Gamma_{0}}^{\text {ob }}$ from a space of ordered based chord diagrams to $R(\Sigma \times I) \otimes \mathbb{Q}$. In the next section we use the map $W_{\mathfrak{g} \int_{0}}^{\mathrm{ob}}$ together with a universal Vassiliev invariant $Z_{\Sigma \times I}^{\mathrm{ob}}$ to show that $\nabla$ is well defined. 
Let $G$ be a group. Let $\Gamma=S^{1 \amalg l}$ be a disjoint union of $l$ oriented circles. A $G$-labeled ordered based chord diagram $D$ with support $\Gamma$ consists of a finite set $S=A \amalg B \amalg C$ of mutually distinct points on $\Gamma$, such that

- on each circle lies exactly one element of $B$ called basepoint, the set $B$ is linearly ordered;

- $C$ is partitioned into subsets of cardinality two called chords, and

- to each point $p$ in $A$ there is an assigned element of the group $G$ called label of $p$.

Usually, we call $G$-labeled ordered based chord diagrams simply chord diagrams. We consider 2-chord diagrams $D$ and $D^{\prime}$ with support $\Gamma$ and $\Gamma^{\prime}$, respectively, as being equal, if there exists a homeomorphism between $\Gamma$ and $\Gamma^{\prime}$ that preserves all additional data. Define the degree $\operatorname{deg}(D)$ of a chord diagram $D$ as the number of its chords.

We represent a chord diagram graphically as follows: the circles of the 1-manifold $\Gamma$ are oriented counterclockwise in the pictures. We connect the two endpoints of a chord by a thin line. The labels of a chord diagram are represented by marking the points of $A$ by a dot on a circle and by writing the labels close to these marked points. The basepoints are marked by the symbol $\times$ on the circle. We draw the basepoints from the left to the right in increasing order, or we label the basepoints by elements of an ordered set. An example of a picture of a $\mathbb{Z}$-labeled chord diagram $D$ of degree 5 is shown in Figure 7.1.

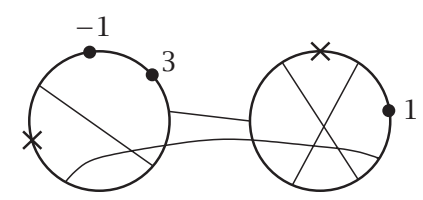

FigURE 7.1. A picture of a $\mathbb{Z}$-labeled chord diagram.

In the following definitions we define vector spaces by generators and graphical relations. We use the convention that all diagrams in a graphical relation coincide everywhere except for the parts we show, and that all configurations of the hidden parts are possible.

DefinITION 7.1. Let $G$ be a group, and $\sigma: G \rightarrow \mathbb{Z} / 2$ a homomorphism of groups. Define $\mathscr{A}^{\mathrm{ob}}(G, \sigma)$ to be the graded $\mathbb{Q}$-vector space generated by $G$-labeled ordered based chord diagrams $D$ modulo relations (4T), ( $\sigma$-Nat), (Rep), (Bas), and (Ord) below.

The 4-term relation (4T):

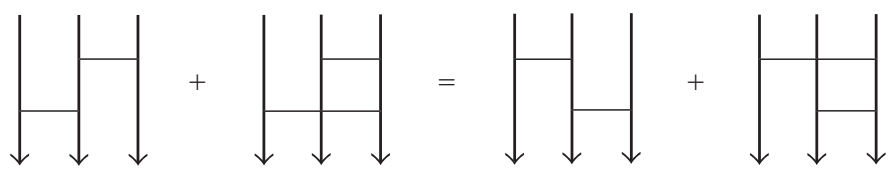

Relation ( $\sigma$-Nat):
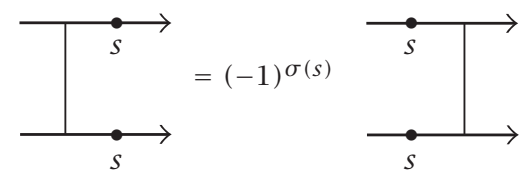
Relations (Rep):

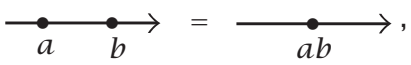

$$
\longrightarrow \text { 늘 }
$$

where $e$ is the neutral element of $G$, and $a b$ denotes the product of $a$ and $b$ in the group $G$.

Relations (Bas):

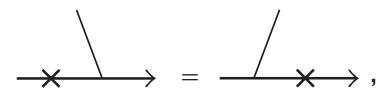

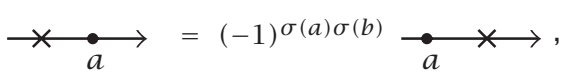

where the element $b \in G$ is defined as the product of the labels on the hidden part of the shown component (the order of the multiplication is not important).

Relation (Ord):
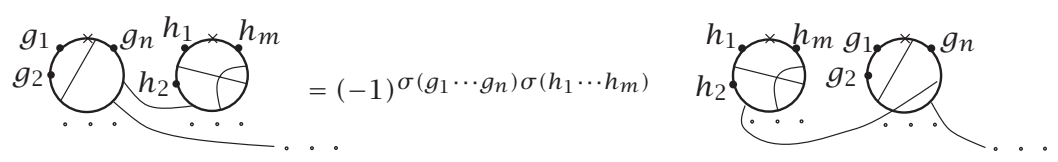

meaning that the change of the order of two neighbored circles gives the sign contribution $(-1)^{\sigma(a) \sigma(b)}$, where $a$ and $b$ are the products of the labels on the two circles.

Definition 7.2. Let $\mathscr{A}^{\mathrm{ob}}(G, \sigma)$ be the quotient of $\mathscr{A}^{\mathrm{ob}}(G, \sigma)$ by the framing independence relation (FI):

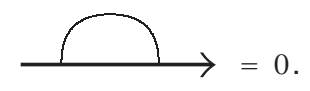

Extending the order of the basepoints of chord diagrams $D_{1}, D_{2}$ to the disjoint union $D_{1} \amalg D_{2}$, by requiring that all basepoints of $D_{1}$ are smaller than those of $D_{2}$, induces a multiplication on $\mathscr{A}(G, \sigma)$ turning it into a graded ring. If $G=\pi_{1}(M)$ and $\sigma: \pi_{1}(M) \rightarrow \mathbb{Z} / 2$ is the first Stiefel-Whitney class of $M$, then we have an isomorphism of rings $\mathscr{C}_{0}(M) \otimes \mathbb{Q} \cong \mathscr{A}_{0}(G, \sigma)$, where $\mathscr{A}_{0}(G, \sigma)$ is the degree-0 part of $\mathscr{A}(G, \sigma)$.

Let $D$ be a picture of a chord diagram such that the basepoint is the highest point on each circle and all labels lie on a horizontal line. An example is shown in Figure 7.2.

We used the order of the oriented circles and the basepoints to draw Figure 7.2. Now we forget this data. We replace each chord as shown in Figure 7.3.

The result is an immersion of labeled circles into the plane. By a homotopy this immersion can be rearranged to a standard embedding, meaning that all labels lie on a horizontal line, no parts of the circles lie below this line, and the projections of the circles to the horizontal line are disjoint. As an example, after having replaced the chords of the diagram in Figure 7.2, we can rearrange the immersion to a standard embedding as shown in Figure 7.4.

During the homotopy we count the number $m$ of times the order of the projections of two labels $s$, s' with $\sigma(s)=\sigma\left(s^{\prime}\right)=1$ to the horizontal line changes. For the example in Figures 7.2 and 7.4, we have $(-1)^{m}=(-1)^{\sigma(b) \sigma(c f g)+\sigma(d) \sigma(f g h)+\sigma(f) \sigma(g)}$. The chosen order of the circles and the chosen highest points of the circles in a standard embedding of labeled circles determine an ordered based chord diagram (without chords) that we call $D^{\prime}$ for the moment. 


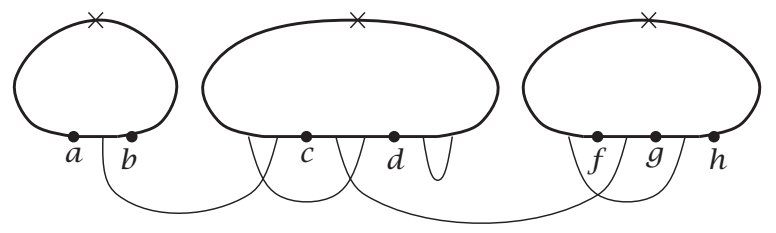

FIGURE 7.2. A special picture of a chord diagram.

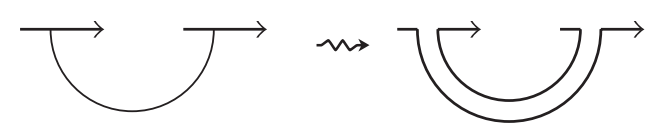

FIGURE 7.3. Replacing a chord.

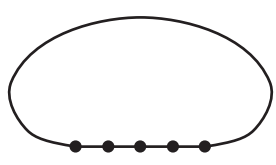

a c g $f$ b

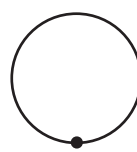

$h$

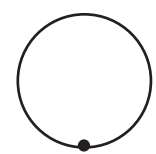

d

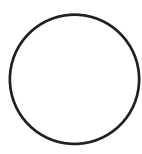

FIGURE 7.4. A standard embedding of labeled circles.

Proposition 7.3. The linear map

$$
W_{\mathfrak{g} \Gamma}^{\mathrm{ob}}: \mathscr{A}^{\mathrm{ob}}(G, \sigma) \longrightarrow \mathscr{A}_{0}^{\mathrm{ob}}(G, \sigma)
$$

that sends a G-labeled ordered based chord diagram $D$ to $(-1)^{m} D^{\prime}$ is well defined.

PRoof. Changing the order of two circles or pushing a label around a circle in a standard embedding of labeled circles as in Figure 7.4 gives the same sign contribution to $(-1)^{m}$ and to $D^{\prime}$. So $W_{\mathrm{g} f}^{\mathrm{ob}}$ is well defined for chord diagrams. We have to verify that $W_{\mathrm{g} f}^{\mathrm{ob}}$ respects the defining relations of $\mathscr{A}^{\mathrm{ob}}(G, \sigma)$.

The map $W_{\mathrm{gf}}^{\text {ob }}$ respects the relation shown in Figure 7.5, where any order of the shown parts of the diagram is possible, because we can slide one thickened chord as in Figure 7.3 along the other one (see [5, Section 3]). This relation implies relation (4T).

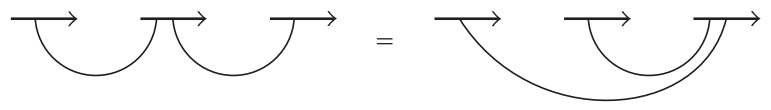

FIGURE 7.5. A 2-term relation.

We can easily verify the compatibility of $W_{\mathfrak{g f}}^{\mathrm{ob}}$ with relations (Rep), (Bas), and (Ord). The compatibility of $W_{\mathfrak{g f}}^{\text {ob }}$ with relation ( $\sigma$-Nat) follows from Figure 7.6, which shows an equation between the elements of $\mathscr{A}_{0}^{\mathrm{ob}}(G, \sigma)$ defined by immersed labeled circles as in the definition of $W_{\mathfrak{g} \int}^{\text {ob }}$.

The formula in Figure 7.6 holds because when we push labels $s$ to their new positions, then in the projection to the horizontal line they are commuted with the same 


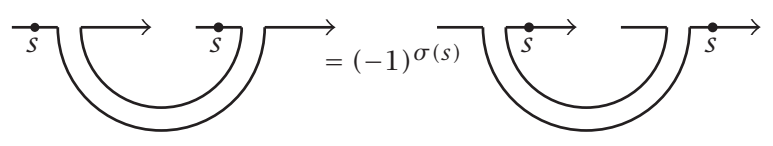

FIGURE 7.6. The compatibility of $W_{\mathfrak{g f}}^{\text {ob }}$ with relation $(\sigma$-Nat).

labels from the remaining part of the diagram, and the commutation between the $s$ on the right side with the $s$ on the left side gives the sign contribution $(-1)^{\sigma(s)}$.

Let $p: A_{0}\left(\pi_{1}(\Sigma), \sigma\right) \rightarrow R(\Sigma \times I) \otimes \mathbb{Q}$ be the homomorphism of rings induced by mapping a circle with a single mark $g$ to $\bar{K}_{g}$. Replacing the chords in a relation (FI) as in Figure 7.3 produces a circle without labels together with at least one additional component. This implies the following corollary.

COROLlaRY 7.4. For nonorientable surfaces $\Sigma$, the map $p \circ W_{\mathfrak{g} f}^{\mathrm{ob}}$ descends to a map

$$
W_{\mathfrak{g}_{0}}^{\mathrm{ob}}: \overline{\mathcal{A}}\left(\pi_{1}(\Sigma), \sigma\right) \longrightarrow R(\Sigma \times I) \otimes \mathbb{Q} .
$$

The reason for the notation $W_{\mathfrak{g} f^{\mathrm{ob}}}^{\mathrm{b}}$ and $W_{\mathfrak{g} \int_{0}}^{\mathrm{ob}}$ is given in [16, Section 1.17], where $W_{\mathfrak{g} \mathfrak{f}}^{\mathrm{ob}}$ and $W_{\mathfrak{g} f_{0}}^{\mathrm{ob}}$ are related to weight systems associated with the Lie superalgebra $\mathfrak{g} \mathfrak{l}(V)$ of endomorphisms of a $\mathbb{Z} / 2$-graded vector space $V$. In the case of $W_{\mathfrak{g} f_{0}}^{\text {ob }}$ the superdimension of $V$ is 0 . We make use of this relation in Section 9.

The map $W_{\mathrm{gf}}^{\text {ob }}$ can be described recursively as follows. For chord diagrams $D$ of degree 0 we have $W_{\mathfrak{g f}}^{\mathrm{ob}}(D)=D$. If $D$ is a chord diagram with at least one chord $c$, then we can use relations (Bas) and (Ord) such that $D$ looks like one of the two diagrams to the left of the symbol $m$ in Figure 7.7.
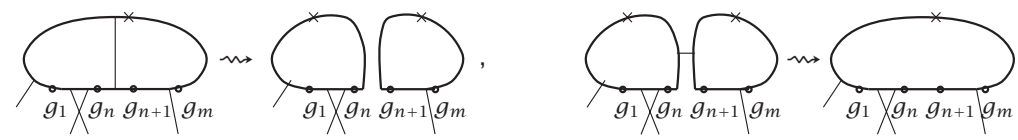

FIGURE 7.7. A recursive description of $W_{\mathfrak{g} \mathfrak{o b}}^{\mathrm{ob}}$.

In Figure 7.7 only the chord $c$ of $D$ is replaced as shown in Figure 7.3. We obtain a chord diagram $D(c)$ with $\operatorname{deg}(D(c))=\operatorname{deg}(D)-1$ and with

$$
W_{\mathfrak{g} \mathfrak{f}}^{\mathrm{ob}}(D)=W_{\mathfrak{g} \mathfrak{l}}^{\mathrm{ob}}(D(c)) .
$$

We can express the recursion formula for $W_{\mathfrak{g f}}^{\text {ob }}$ from Figure 7.7 without using embeddings by the local replacement rules involving basepoints as shown in Figure 7.8.

8. Proof of Theorems 4.1 and 4.2. Let $\Sigma=\bigcup_{i=0}^{k} B_{i}$ be a decomposed surface or let $\Sigma=P^{2}$. Let $L$ be a link in $\Sigma \times I$. Recall the definition of the universal Vassiliev invariant $Z_{\Sigma \times I}(L)$ from [15]. For a decomposed surface $\Sigma$, this definition is roughly as follows: after applying an isotopy we can assume that $L \cap B_{i} \times I$ is in a standard position for all $i>0$. The tangle $L \cap B_{0} \times I$ is turned into a nonassociative tangle $T$ by 


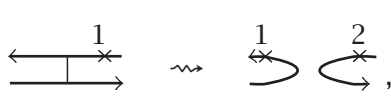

or

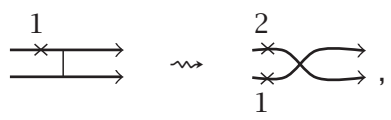

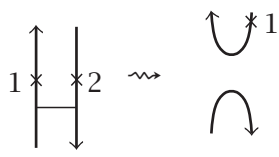

or

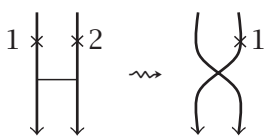

FIGURE 7.8. Descriptions of $W_{\mathfrak{g} \int^{\mathrm{ob}}}^{\mathrm{b}}$ and $W_{\mathfrak{g} \int_{0}}^{\mathrm{ob}}$ by local replacement rules.

choosing a bracketing on the ordered set $L \cap\left(\partial B_{0}\right) \times I$ satisfying some conditions. Then $Z_{\Sigma \times I}(L)$ is obtained from the universal Vassiliev invariant $Z(T)$ of the nonassociative tangle $T$ (see $[4,14]$ ) by gluing labeled intervals to those pairs of boundary points of diagrams in $Z(T)$ whose corresponding boundary points of $T$ are connected by an interval in $L \cap\left(\Sigma \backslash B_{0}\right) \times I$.

Let $\mathscr{L}^{\mathrm{ob}}(M)$ be the set of isotopy classes of ordered based links in $M$. Let $\hat{\mathscr{A}}^{\mathrm{ob}}(M)$ be the graded completion of $\overline{\mathscr{A}}^{\mathrm{ob}}(M)=\overline{\mathscr{A}}^{\mathrm{ob}}\left(\pi_{1}(M), \sigma\right)$. Define a map,

$$
Z_{\Sigma \times I}^{\mathrm{ob}}: \mathscr{L}^{\mathrm{ob}}(\Sigma \times I) \longrightarrow \hat{A}^{\mathrm{ob}}(\Sigma \times I),
$$

by equipping the chord diagrams in the series $Z_{\Sigma \times I}(L)$ with an order and with basepoints according to the order and the basepoints of $L$. The map $Z_{\Sigma \times I}^{\mathrm{ob}}$ is well defined because the chord endpoints commute with the basepoints by relation (Bas) (see $[15$, Lemma 25]). Let $\hat{R}(M):=(R(M) \otimes \mathbb{Q})[[h]]$. Define

$$
\widehat{W}_{\mathfrak{g} \int_{0}}^{\mathrm{ob}}: \hat{\mathcal{A}}^{\mathrm{ob}}(G, \sigma) \rightarrow \widehat{R}(\Sigma \times I)
$$

by extending $\widehat{W}_{\mathrm{g}_{0}}^{\mathrm{ob}}(D)=W_{\mathrm{g} \Gamma_{0}}^{\mathrm{ob}}(D) h^{\operatorname{deg} D}$ for any chord diagram $D$ to formal power series. Finally, define the invariant $\hat{\nabla}$ of ordered based links by

$$
\hat{\nabla}=\widehat{W}_{\mathfrak{g} \Upsilon_{0}}^{\mathrm{ob}} \circ Z_{\Sigma \times I}^{\mathrm{ob}} .
$$

Turn $\hat{R}(M)$ into a $\mathbb{Z}[z]$-module by $z \cdot a:=\left(e^{h / 2}-e^{-h / 2}\right) a$ for $a \in \hat{R}(M)$.

LEMmA 8.1. The invariant $\hat{\nabla}$ of ordered based links induces $a \mathbb{Z}[z]$-linear map

$$
\begin{gathered}
\hat{\nabla}: \mathscr{C}^{\#}\left(\sum \times I\right) \longrightarrow \hat{R}\left(\sum \times I\right) \text { satisfying } \\
\hat{\nabla}(L) \equiv \bar{L} \bmod h \quad \text { for all ordered based links } L .
\end{gathered}
$$

Proof. Let $\left(L_{+}, L_{-}, L_{\|}\right)$be a skein triple of ordered based links. Let $B_{1}=\downarrow \downarrow 1$ and $B_{2}=\Downarrow 1 \downarrow 2$. Define the composition of diagrams $D_{1}, D_{2}$ by placing $D_{1}$ onto the top of $D_{2}$. In the recursive description of $W_{\mathfrak{g} f}^{\text {ob }}$ (see Figure 7.8) a chord $B_{i} \circ \downarrow \downarrow$ between two

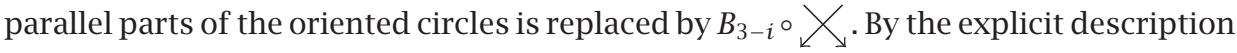


of $Z_{\Sigma \times I}^{\mathrm{ob}}$ and by (7.9) we have, for some $i \in\{1,2\}$,

$$
\begin{aligned}
& \hat{\nabla}\left(L_{+}\right)-\hat{\nabla}\left(L_{-}\right) \\
& =\widehat{W}_{\mathfrak{g} \Gamma_{0}}^{\mathrm{ob}}\left(B_{i} \circ \exp (\downarrow \downarrow / 2) \circ \searrow \searrow\right)-\widehat{W}_{\mathfrak{g} \Gamma_{0}}^{\mathrm{ob}}\left(B_{i} \circ \exp (-\downarrow \downarrow \downarrow / 2) \circ \searrow \searrow\right. \\
& =\sum_{n=0}^{\infty} \frac{1}{4^{n}(2 n+1) !} \widehat{W}_{\mathfrak{g} \mathfrak{f}_{0}}^{\mathrm{ob}}\left(B_{i} \circ \downarrow \downarrow^{2 n+1} \circ \searrow_{\searrow}\right) \\
& =\sum_{n=0}^{\infty} \frac{h^{2 n+1}}{4^{n}(2 n+1) !} \widehat{W}_{\mathfrak{g} \Gamma_{0}}^{\mathrm{ob}}\left(B_{3-i}\right)=\left(e^{h / 2}-e^{-h / 2}\right) \hat{\nabla}\left(L_{\|}\right)=z \cdot \hat{\nabla}\left(L_{\|}\right) \text {. }
\end{aligned}
$$

It is obvious by relations (Ord) and (Bas) for ordered based links and chord diagrams that $\hat{\nabla}$ is well defined on $\mathscr{C}(\Sigma \times I)$. Since $\hat{R}(\Sigma \times I)$ has no nontrivial torsion elements as a $\mathbb{Z}[z]$-module, $\hat{\nabla}$ descends to $\mathscr{C}^{\#}\left(\sum \times I\right)$. Equation (8.4) follows because for ordered based links $L$ whose $i$ th component is homotopic to $w_{i} \in \pi_{1}(\Sigma \times I)$ we have $Z_{\Sigma \times I}^{\mathrm{ob}}(L) \equiv$ $D_{L} \bmod h$, where $D_{L} \in \bar{A}\left(\sum \times I\right)_{0}$ is a product of $r$ oriented circles with a single label $w_{i}$, and $W_{\mathfrak{g}_{0}}^{\mathrm{ob}}\left(D_{L}\right)=\pi\left(D_{L}\right)=\bar{L} \in R\left(\sum \times I\right) \otimes \mathbb{Q}$.

It remains to make a change of parameters to show that the Conway polynomial is well defined.

Proof of TheOrem 4.1. (1) The uniqueness of $\nabla$ follows because the $\mathbb{Z}[z]$ module $\mathbb{Z}[z] \otimes R(\Sigma \times I)$ has a trivial torsion submodule and the condition $\nabla(L)=\bar{L}$ for descending links $L$ with $\theta(L) \neq 0$ prescribes the image of the generators $\mathscr{G}_{\Sigma}$ of $\mathscr{C}^{\#}(\Sigma \times I)$ (see Proposition 6.4). We prove the existence of $\nabla$. Proposition 2.4 implies that the set $\overline{\mathscr{G}}_{\Sigma}=\left\{\bar{L} \mid L \in \mathscr{G}_{\Sigma}\right\}$ is a basis of the $\mathbb{Z}$-module $R(\Sigma \times I)$. Define a $\mathbb{Z}[z]$-linear map $\kappa: \mathbb{Z}[z] \otimes_{\mathbb{Z}} R(\Sigma \times I) \rightarrow \mathscr{C}^{\#}(\Sigma \times I)$ on basis elements by

$$
\kappa\left(1 \otimes \bar{K}_{w_{1}} \cdots \bar{K}_{w_{n}}\right):=K_{w_{1}} \cdots K_{w_{n}} .
$$

The map $\kappa$ is surjective. Consider the composition

$$
\mathbb{Z}[z] \otimes_{\mathbb{Z}} R(\Sigma \times I) \stackrel{\kappa}{\longrightarrow} \mathscr{C}^{\#}(\Sigma \times I) \stackrel{\hat{\nabla}}{\longrightarrow} \hat{R}(\Sigma \times I) .
$$

By Lemma 8.1 we have $\hat{\nabla}(\kappa(\bar{L})) \equiv \bar{L} \bmod h$ for all ordered based links $L$. This implies that $\bar{G}_{\Sigma}$ is mapped injectively to the basis $\hat{\nabla}\left(\kappa\left(\bar{G}_{\Sigma}\right)\right) \bmod h$ of the $\mathbb{Q}$-vector space $\hat{R}(\Sigma \times$ $I) /(h)$. Considering inductively the sets $\hat{\nabla}\left(\kappa\left(\bar{\varphi}_{\Sigma}\right)\right) \bmod h^{n}$ we see that $\hat{\nabla}\left(\kappa\left(\bar{\varphi}_{\Sigma}\right)\right)$ is linearly independent over $\mathbb{Q}[z]$ (where $z \cdot a=\left(e^{h / 2}-e^{-h / 2}\right) a$ ). This implies that $\hat{\nabla} \circ$ $\kappa: \mathbb{Z}[z] \otimes R(\Sigma \times I) \rightarrow \hat{R}(\Sigma \times I)$ is injective. Hence $\kappa$ is an isomorphism and the map $\kappa^{-1}: \mathscr{C}^{\#}\left(\sum \times I\right) \rightarrow \mathbb{Z}[z] \otimes R\left(\sum \times I\right)$ has the property $\kappa^{-1}(L)=\bar{L}$ for all $\hat{\mathscr{S}}$-descending links $L$ with $\theta(\bar{L}) \neq 0$. We obtain $\nabla$ as the composition of the canonical projection $\mathscr{C}(\Sigma \times I) \rightarrow \mathscr{C}^{\#}(\Sigma \times I)$ with $\kappa^{-1}$.

Proof OF TheOrem 4.2. (1) Let $\Sigma$ be the Möbius strip $X$. Let $L \subset X \times I$ be a $\hat{\mathscr{Y}}$ descending link. If $\theta(\bar{L})=0$, then $L=0 \in \mathscr{C}^{\#}(X \times I)$ by Lemma 6.1. Theorem 4.1 implies that $\nabla(L)=0$ because $\nabla$ factors through $\mathscr{C}^{\#}(X \times I)$.

It follows easily from Lemma 6.1 and Theorem 4.1 that $\nabla\left(L_{1} L_{2}\right)=\nabla\left(L_{1}\right) \nabla\left(L_{2}\right)$ for all $\hat{\mathscr{S}}$-descending links $L_{1}, L_{2} \subset X \times I$. Lemma 5.1 implies this formula for all ordered based links $L_{1}, L_{2} \subset X \times I$. 
The case $\Sigma=P^{2}$ of the theorem can be deduced from the case $\Sigma=X$. For $\Sigma=I^{2}$ and $\Sigma=S^{1} \times I$ part (1) of the theorem is trivial.

(2) It is enough to show that $L=(-1)^{|L|_{0}} L^{*} \in \mathscr{C}^{\#}(\Sigma \times I)$ for $\Sigma=X$. Let $L$ be a diagram of an ordered based link in $X \times I$. Let $k_{1}(L)$ be the number of crossings of $L$ and let $k_{2}(L)$ be the minimal number of crossing changes needed to pass from $L$ to a diagram of an $\hat{\mathscr{S}}$-descending link. We prove the theorem by induction on the lexicographical order on $\left(k_{1}(L), k_{2}(L)\right)$.

Assume that $k_{2}(L)=0$. Then $L=K_{i_{1}} \cdots K_{i_{r}}$ is a product of descending knots. By Lemma 6.1 we have $L=0=L^{*}$ if $|L|_{0}>0$. Assume that $|L|_{0}=0$. Then Lemmas 6.1 and 6.2 imply that

$$
\begin{aligned}
K_{i_{1}} \cdots K_{i_{r}} & =(-1)^{r(r-1) / 2} K_{i_{r}} \cdots K_{i_{1}}=(-1)^{r(r-1) / 2} K_{i_{r}}^{*} \cdots K_{i_{1}}^{*} \\
& =\left(K_{i_{1}} \cdots K_{i_{r}}\right)^{*}=(-1)^{|L|_{0}}\left(K_{i_{1}} \cdots K_{i_{r}}\right)^{*} .
\end{aligned}
$$

If $k_{1}(L)=0$, then we also have $k_{2}(L)=0$ and we are back in the first case. Now let $k_{1}(L)>0$ and $k_{2}(L)>0$. Choose a crossing of $L$ such that $k_{2}\left(L_{1}\right)=k_{2}(L)-1$, where $L_{1}$ is obtained from $L$ by changing this crossing and let $L_{2}$ be obtained by splicing the crossing. Then for an $\epsilon \in\{ \pm 1\}$ we have, by induction,

$$
\begin{aligned}
L & =L_{1}+\epsilon z L_{2}=(-1)^{\left|L_{1}\right|_{0}} L_{1}^{*}+\epsilon z(-1)^{\left|L_{2}\right|_{0}} L_{2}^{*} \\
& =(-1)^{|L|_{0}}\left(L_{1}^{*}-\epsilon z L_{2}^{*}\right)=(-1)^{|L|_{0}} L^{*} .
\end{aligned}
$$

In this computation we used that, for a skein triple $\left(L_{+}, L_{-}, L_{\|}\right)$of links, we have $\left|L_{+}\right|_{0}=$ $\left|L_{-}\right|_{0}=\left|L_{\|}\right|_{0} \pm 1$ and $\left(L_{-}^{*}, L_{+}^{*}, L_{\|}^{*}\right)$ is also a skein triple.

9. Coverings and the Conway polynomial. Let $\bar{A}(G, \sigma)$ be the vector space generated by $G$-labeled chord diagrams (without order and basepoints) modulo relations (4T), $(\sigma$-Nat), (Rep), and (FI) (see Section 7). For a 3-manifold $M$ we define $\bar{A}(M):=$ $\bar{A}\left(\pi_{1}(M), \sigma\right)$ where $\sigma$ is the orientation character of $M$. The universal Vassiliev invariant $Z_{\Sigma \times I}$ (see [15]) takes values in a completion of $\overline{\mathcal{A}}(\Sigma \times I)$. For a labeled diagram $D$, define

$$
\sigma(D)=\sum \sigma(g) \in \mathbb{Z} / 2,
$$

where the sum runs over all labels $g$ on the skeleton of $D$.

Recall a result of [15] for the special case of the 2-fold covering $p: S^{2} \times I \rightarrow P^{2} \times$ I. For a chord diagram $D$ labeled by the elements of $\pi_{1}\left(P^{2}, *\right) \cong \mathbb{Z} / 2$, we omit the points labeled by the neutral element in our pictures. The points of $D$ labeled by the nontrivial element are simply represented by a dot (without label). There exists a map $p^{*}: \bar{A}\left(P^{2} \times I\right) \rightarrow \bar{A}\left(S^{2} \times I\right)$ defined by the replacement rules shown in (9.2),

$$
p^{*}(\downarrow)=\bigvee, \quad p^{*}(\downarrow \downarrow)=\downarrow \downarrow \downarrow \downarrow-\downarrow \downarrow \downarrow \downarrow \downarrow .
$$

For a link $L \subset P^{2} \times I$, we have

$$
p^{*} \circ Z_{P^{2} \times I}(L)=Z_{S^{2} \times I}\left(p^{-1}(L)\right)
$$


(see [15, Theorem 4, part (2)]). Links in $S^{2} \times I$ are in a natural bijection with links in $S^{3}$ or $\mathbb{R}^{3}$, and $Z_{S^{2} \times I}$ corresponds to the usual Kontsevich integral under this bijection ([15, Theorem 4, part (1)]).

Comparing $\bar{A}\left(P^{2} \times I\right)$ with $\bar{A}^{\text {ob }}\left(P^{2} \times I\right)$ we remark huge differences: elements of odd degree in $\bar{A}\left(P^{2} \times I\right)$ are 0 (see [15, proof of Corollary 4]), whereas this is not true for $\bar{A}^{\mathrm{ob}}\left(P^{2} \times I\right)$ (otherwise the recursive description in Figure 7.8 of the nontrivial map $W_{\mathfrak{g f}_{0}}^{\mathrm{ob}}$ could not exist). However, for ordered based diagrams $D$ on a single circle with $\sigma(D)=1$, the order and basepoints are superfluous and the difference between $\bar{A}\left(P^{2} \times I\right)$ and $\overline{\mathscr{A}}^{\mathrm{ob}}\left(P^{2} \times I\right)$ vanishes for these special diagrams.

As for usual chord diagrams there are many descriptions of spaces isomorphic to $\bar{A}(G, \sigma)$. By $G$-labeled trivalent diagrams we mean usual trivalent diagrams $D$ (see [3]) together with a distinguished subset of points on edges of $D$ that are equipped with local orientations and labeled by the elements of $G$. For points on the skeleton of $D$ we assume that the local orientation coincides with the orientation of the skeleton. In particular, labeled chord diagrams are labeled trivalent diagrams. The space $\bar{A}(G, \sigma)$ is isomorphic to the $\mathbb{Q}$-vector space generated by trivalent diagrams modulo relations (FI), (Rep) (see Section 7), (STU), (IHX), (AS) (see [3]) and modulo relations (Comult), and (Inv1) shown below

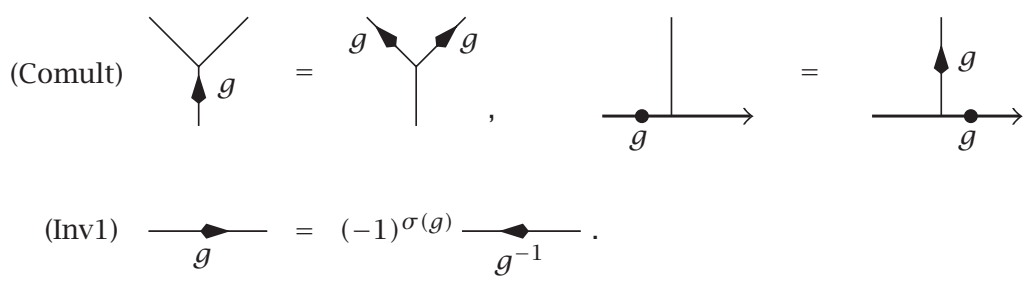

This can be proven along the same lines as in [3] (with a slight difference in the case where an internal trivalent vertex connects to the skeleton of the diagram via two edges). A similar description by generators and relations exists for $\overline{\mathcal{A}}^{\mathrm{ob}}(G, \sigma)$. The space $\bar{A}(G, \sigma)$ is a coalgebra with comultiplication $\Delta$ given by

$$
\Delta(D)=\sum_{D=D_{1} \cup D_{2}} D_{1} \otimes D_{2}
$$

where the sum runs over all trivalent diagrams $D_{1}, D_{2} \subset D$, such that $D \backslash \Gamma=\left(D_{1} \backslash \Gamma\right) \amalg$ $\left(D_{2} \backslash \Gamma\right)$. Beside the comultiplication $\Delta$, there exists a map

$$
\Delta^{\mathrm{ob}}: \bar{A}(G, \sigma) \rightarrow \bar{A}^{\mathrm{ob}}(G, \sigma) \otimes \bar{A}^{-\mathrm{ob}}(G, \sigma)
$$

defined by the same formula as in (9.5), where this time $D_{1}$ and $D_{2}$ are equipped with the order and basepoints that are arbitrarily chosen for the first diagram and copied to the second.

Now consider the $\mathbb{Z} / 2$-labeled trivalent diagrams $D$ with skeleton $\Gamma$ where $D \backslash \Gamma$ is connected and all the labeled points lie close to univalent vertices of $D \backslash \Gamma$. Define 
diagrams $p_{1}(D)$ and $p_{2}(D)$ by the local replacement rules shown in (9.7),

$$
\begin{gathered}
p_{i}(\downarrow)=\bigvee, \quad p_{i}(Y)=Y, \\
\left.p_{1}(\downarrow)=p_{2}(\downarrow)=\downarrow \downarrow \downarrow \downarrow\right)=p_{2}(\downarrow)=\downarrow \downarrow \downarrow
\end{gathered}
$$

For diagrams $D$ as above it is easy to see that $p^{*}$ satisfies the equation

$$
p^{*}(D)=p_{1}(D)+(-1)^{\operatorname{deg} D} p_{2}(D) .
$$

For diagrams $D$ where each of the $c>1$ connected components of $D \backslash \Gamma$ satisfies the conditions above, $p^{*}(D)$ can be expressed as a sum with $2^{c}$ terms by extending (9.8) multilinearly over these connected components. Using relations (Comult) and (Inv1), we can apply this formula whenever $D \backslash \Gamma$ is a forest.

We call a connected component of a diagram $D \backslash \Gamma$ a wheel with (resp., without) a dot, if it looks like the left (resp., right) side of Figure 9.1.
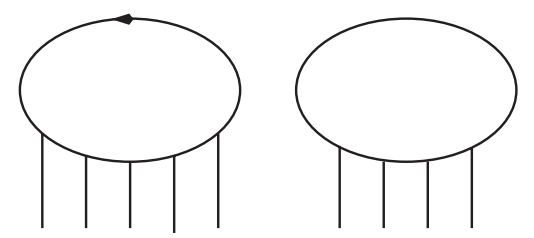

FigURE 9.1. A wheel with and without a dot.

As a consequence of (9.8) we can construct many elements in $\operatorname{Ker}\left(p^{*}\right)$. An example is given in the following lemma.

LEMMA 9.1. Let $D$ be a trivalent diagram such that one component of $D \backslash \Gamma$ is a wheel with a dot. Then $p^{*}(D)=0$.

Proof. Applying the (STU) relation to the wheel with a dot we obtain the two diagrams $D_{1}$ and $D_{2}$ shown in Figure 9.2.

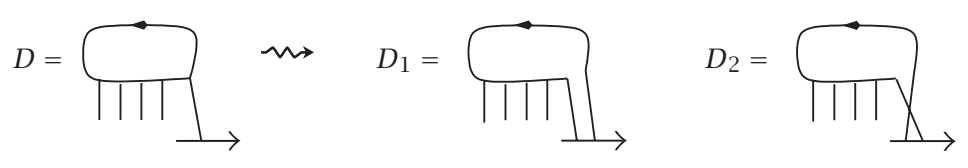

FIGURE 9.2. $D=D_{1}-D_{2}$ by the (STU) relation.

By (9.8) we then have

$$
\begin{aligned}
p^{*}(D) & =p^{*}\left(D_{1}-D_{2}\right) \\
& =p_{1}\left(D_{1}\right)+(-1)^{\operatorname{deg}\left(D_{1}\right)} p_{2}\left(D_{1}\right)-p_{1}\left(D_{2}\right)-(-1)^{\operatorname{deg}\left(D_{2}\right)} p_{2}\left(D_{2}\right)=0
\end{aligned}
$$

because $p_{i}\left(D_{1}\right)=p_{i}\left(D_{2}\right)$. 
Consider an interval $J$ on the skeleton $\Gamma=S^{1}{ }^{\amalg \ell}$ of a diagram $D$. Cutting $D$ at the endpoints of $J$, we obtain a diagram $D^{\prime}$ with skeleton $S^{1^{\amalg \ell-1}} \amalg J \amalg\left(S^{1} \backslash J\right)$. When $D^{\prime}$ is

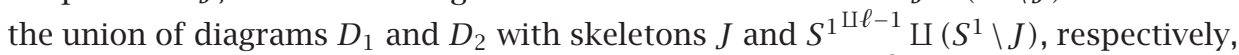
then we say that $D_{1}$ is an isolated part of $D$. The map $W_{\mathrm{gf}_{0}}^{\mathrm{ob}}: \bar{A}^{-\mathrm{ob}}\left(P^{2} \times I\right) \rightarrow R\left(P^{2} \times I\right) \otimes \mathbb{Q}$ has the following special property.

LEMMA 9.2. Let $D$ be an ordered based $\mathbb{Z} / 2$-labeled diagram such that $\sigma(D)=0$, or the number of circles of the skeleton of $D$ plus the degree of $D$ is even, or $D$ contains an isolated part of odd degree. Then $W_{\mathfrak{g} f_{0}}^{\mathrm{ob}}(D)=0$.

The proof of the lemma follows easily by expanding $W_{\mathfrak{g} \int_{0}}^{\mathrm{ob}}(D)$ as a linear combination of multiples of $\bar{K}_{e}=0$ and $\bar{K}_{s}^{2}=0 \in R\left(P^{2} \times I\right) \otimes \mathbb{Q}$.

Some properties of $W_{\mathfrak{g f}_{0}}^{\text {ob }}$ for ordered based $\mathbb{Z} / 2$-labeled diagrams can more conveniently be proven using the Lie superalgebra $\mathfrak{g} \mathfrak{l}(1 \mid 1)$. We identify $\mathfrak{g} \mathfrak{f}(1 \mid 1)$ with $2 \times 2$-matrices using a homogeneous basis of the defining (1|1)-dimensional representation of $\mathfrak{g} \mathfrak{l}(1 \mid 1)$. The rough idea, how the usual description of the weight system associated to $\mathfrak{g} \mathfrak{f}(1 \mid 1)$ (see [22]) extends to $\mathbb{Z} / 2$-labeled diagrams is by mapping a dot on the skeleton to the morphism $\tau$ in (9.10),

$$
\downarrow \longmapsto\left(\begin{array}{ll}
0 & 1 \\
1 & 0
\end{array}\right)=\tau
$$

(cf. [16, Section 1.17], recall from there how labels can influence signs). Given an ordered based $\mathbb{Z} / 2$-labeled diagram $D$ with $\sigma(D)=1$, we cut the first circle of the skeleton of $D$ at its basepoint. Then the construction of weight systems maps the resulting diagram to a morphism of the form $\lambda \cdot \tau$. It is easy to show that $W_{\mathfrak{g} \int_{0}}^{\mathrm{ob}}(D)=\lambda \bar{K}_{s}$. In particular, the number $\lambda=: W^{s}(D)$ depends only on $D \in \bar{A}^{\mathrm{ob}}\left(P^{2} \times I\right)$.

Define $W^{e}: \bar{A}\left(S^{2} \times I\right) \rightarrow \mathbb{Q}$ by $W^{e}(D)=\lambda$ whenever $W_{\mathfrak{g} f_{0}}^{\mathrm{ob}}(D)=\lambda \bar{K}_{e}$. The following properties of $W=W^{e}$ for diagrams without labels are proven in [22]. The diagram on the right side of (9.12) is a trivalent diagram in the sense of this paper (which means it must not contain a connected component that does not connect to the skeleton of the diagram). They extend to $W=W^{s}$ in a straightforward way,

$$
\begin{aligned}
& W(\neg)=W\left(\begin{array}{l}
Y \\
Y
\end{array}\right)=0 \\
& W(\stackrel{\longrightarrow}{\longrightarrow})=W\left(\begin{array}{l}
\longrightarrow \longrightarrow \\
\longrightarrow
\end{array}\right) \\
& w(\downarrow \bigcirc)=-2 W(\downarrow \downarrow) .
\end{aligned}
$$

Equations (9.11), (9.12), and (9.13) for $W=W^{e}, W^{s}$, (9.5), (9.6), (9.8), and the (STU) relation imply the following lemma.

LEMMA 9.3. Equations (9.11), (9.12), and (9.13) also hold for

$$
W=W^{e} \circ p^{*}, \quad W=\left(W^{s} \otimes W^{s}\right) \circ \Delta^{\mathrm{ob}} .
$$


Now we are ready to prove the following important lemma.

LEMмA 9.4. Let $D$ be $a \mathbb{Z} / 2$-labeled diagram with skeleton $S^{1}$ and $\sigma(D)=1$. Then

$$
\left(W^{s} \otimes W^{s}\right) \circ \Delta^{\mathrm{ob}}(D)=W^{e}\left(p^{*}(D)\right) .
$$

Proof. The proof is divided into four steps.

(1) Assume that a component of $D \backslash \Gamma$ is a wheel with a dot. Then $W^{s}(D)=0$ (by (9.12) and Lemma 9.2 it is sufficient to verify this for a degree-2 wheel with a dot. This case follows from a straightforward computation).

(2) We show now that when the lemma holds for $D$, then it also holds for all the diagrams $D^{\prime}$ with $D \subset D^{\prime}$ where $C=D^{\prime} \backslash D$ is connected and $C$ is not a tree. If $C$ is a wheel with a dot, then we have

$$
\left(W^{s} \otimes W^{s}\right) \circ \Delta^{\mathrm{ob}}\left(D^{\prime}\right)=0, \quad W^{e}\left(p^{*}\left(D^{\prime}\right)\right)=0
$$

by part (1) of this proof and Lemma 9.1. If $C$ is not a wheel, then (9.16) follows from Lemma 9.4 and (9.11). If $C$ is a wheel of odd degree, then (9.16) is implied by the (STU) relation, the previous case (where $C$ was not a wheel), and Lemma 9.2. Finally, if $C$ is a wheel of even degree without a dot, then we have $W_{\mathfrak{g} \Gamma_{0}}^{\mathrm{ob}}\left(D^{\prime}\right)=-2 W_{\mathfrak{g} \Gamma_{0}}^{\mathrm{ob}}(D)$ by $(9.12)$ and (9.13), which implies that

$$
\left(W^{s} \otimes W^{s}\right) \circ \Delta^{\mathrm{ob}}\left(D^{\prime}\right)=-4\left(W^{s} \otimes W^{s}\right) \circ \Delta^{\mathrm{ob}}(D)=-4 W^{e}\left(p^{*}(D)\right)=W^{e}\left(p^{*}\left(D^{\prime}\right)\right) .
$$

(3) Now assume that the lemma holds for a diagram $D$ and consider $D \subset D^{\prime}$ such that $C=D^{\prime} \backslash D$ is a tree. We call the tree $C$ a comb if we cannot apply (9.11) to the part $C$ of the diagram $D$. By Lemma 9.3 and (9.12) we only need to consider combs of degrees 1,2 , 3, and 4 . By the (STU) relation and part (2) of this proof, we may arrange the univalent vertices of $C$ on the skeleton $S^{1}$ in any order we want. We then apply relation (Comult) to reduce the configuration of labels we need to consider. In the following we investigate the remaining possibilities:

$\operatorname{deg} C=1$ : by relation (FI) and [16, Lemma 1.9] there is nothing to prove in this case (alternatively, there is a direct proof similar to the case $\operatorname{deg} C=3$ );

$\operatorname{deg} C=2$ : we only need to consider the case shown in (9.18), where we apply relations (STU) and (AS) to reduce this case to part (2) of this proof,

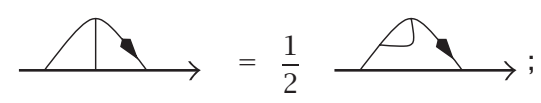

$\operatorname{deg} C=3$ : all possible configurations of $C \subset D^{\prime}$ can be reduced to the case shown in (9.19), where we prove that $p^{*}\left(D^{\prime}\right)=0$

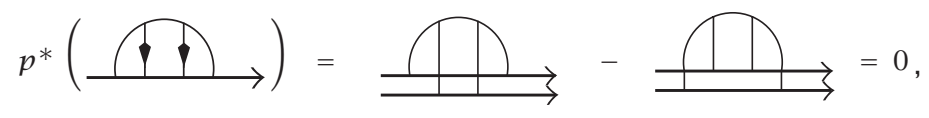

by Lemma 9.2 we have $\left(W^{s} \otimes W^{s}\right) \circ \Delta^{\mathrm{ob}}\left(D^{\prime}\right)=0$ because $\Delta^{\mathrm{ob}}\left(D^{\prime}\right)$ is a linear combination of elements $D_{1} \otimes D_{2}$ such that $D_{1}$ or $D_{2}$ has an isolated part of odd degree; 
$\operatorname{deg} C=4$ : it is sufficient to consider a comb as shown on the left side of (9.20),

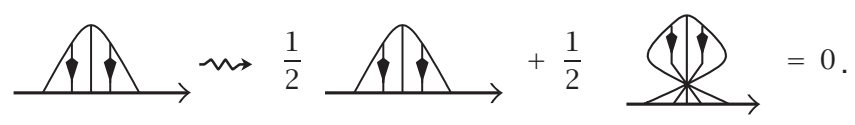

By the (STU) relation and part (2) of this proof, we can equivalently consider the linear combination of two diagrams shown on the right side of (9.20). But by relation (AS) this element equals 0 .

(4) Conclusion: for diagrams of degree 0 the lemma is obvious. Using parts (2) and (3) of this proof, the lemma follows by induction.

It would be interesting to know to what extent Theorem 4.3 can be generalized to links. A counterexample to Lemma 9.4 for chord diagrams on more than one circle is shown in Figure 9.3.

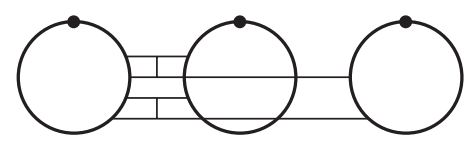

FIGURE 9.3. A diagram $D$ with $\left(W^{s} \otimes W^{s}\right) \circ \Delta^{\mathrm{ob}}(D)=0$ and $W^{e}\left(p^{*}(D)\right) \neq 0$.

Lemma 9.4 is the main ingredient in the following proof of Theorem 4.3.

Proof of Theorem 4.3. Define $\mathbb{Q}[[h]]$-valued maps $\widehat{W}^{e}$ and $\widehat{W}^{s}$ by $\widehat{W}^{e}(D)=$ $W^{e}(D) h^{\operatorname{deg} D}$ and $\widehat{W}^{s}(D)=W^{s}(D) h^{\operatorname{deg} D}$. Let $K^{\mathrm{ob}} \subset P^{2} \times I$ be a based knot with $\sigma(K)=1$ and let $K$ be $K^{\mathrm{ob}}$ without its basepoint. Then we have

$$
\begin{aligned}
\widehat{W}^{e} \circ Z_{S^{2} \times I}\left(p^{-1}(K)\right) & =\widehat{W}^{e} \circ p^{*}\left(Z_{P^{2} \times I}(K)\right) \\
& =\left(\widehat{W}^{s} \otimes \widehat{W}^{s}\right) \circ \Delta^{\mathrm{ob}}\left(Z_{P^{2} \times I}(K)\right) \\
& =\left(\widehat{W}^{s} \circ Z_{P^{2} \times I}^{\mathrm{ob}}\left(K^{\mathrm{ob}}\right)\right)^{2},
\end{aligned}
$$

where the first equality follows from (9.3), the second equality follows from Lemma 9.4 , and the last one follows because $Z_{P^{2} \times I}(K)$ is group-like.

It remains to verify that (9.21) is compatible with the replacement of parameters in the proof of Theorem 4.1; by Proposition 6.4 there exist $f, g \in \mathbb{Z}[z]$ such that

$$
\nabla\left(p^{-1}(K)\right)=g(z) \bar{K}_{e}, \quad \nabla\left(K^{\mathrm{ob}}\right)=f(z) \bar{K}_{s} .
$$

By Lemma 8.1 we have

$$
\begin{aligned}
\widehat{W}^{e} \circ Z_{S^{2} \times I}\left(p^{-1}(K)\right) & =g\left(e^{h / 2}-e^{-h / 2}\right) \widehat{W}^{e} \circ Z_{S^{2} \times I}\left(K_{e}\right), \\
\widehat{W}^{S} \circ Z_{P 2 \times I}\left(K^{\mathrm{ob}}\right) & =f\left(e^{h / 2}-e^{-h / 2}\right) \widehat{W}^{S} \circ Z_{P^{2} \times I}\left(K_{S}\right) .
\end{aligned}
$$

As a simple consequence of the construction of $Z_{P^{2} \times I}$ we have

$$
\left(\widehat{W}^{s} \circ Z_{P^{2} \times I}\left(K_{S}\right)\right)^{2}=\widehat{W}^{e} \circ Z_{S^{2} \times I}\left(K_{e}\right) .
$$

Equations (9.21), (9.22), (9.23), and (9.24) imply that $g(z)=f(z)^{2}$, what we wanted to prove. 
Lemma 9.4 can be generalized from $P^{2}$ to the Möbius strip $X$ using the weight system $W_{\mathrm{g}_{0}}^{\mathrm{ob}} \bmod \left(\bar{K}_{i} \bar{K}_{j} \mid i, j \in \mathbb{Z}\right)$. A generalization of Theorem 4.3 to this case is not straightforward because (9.22) becomes more complicated, and for ordered based links $L$ in the ideal $\left(K_{i} K_{j} \mid i, j \in \mathbb{Z}\right) \subset \mathscr{C}(X \times I)$ we may have

$$
\widehat{W}_{\mathfrak{g} \Upsilon}^{\mathrm{ob}} \circ Z_{X \times I}(L) \not \equiv 0 \bmod \left(\bar{K}_{i} \bar{K}_{j} \mid i, j \in \mathbb{Z}\right)
$$

(e.g., $\left.L=K_{-1} K_{1} K_{3}\right)$.

Appendix. In the following we apply the methods of this paper to determine the Homfly and Kauffman skein modules of cylinders over oriented surfaces with boundary. For the Homfly skein module we rediscover the main result of [18]. Our choice of representatives of conjugacy classes in $\pi_{1}(\Sigma)$ is different from the choice made in [18]. Our choice has the advantage that it allows us to determine the structure of the 2-variable Kauffman skein module with similar methods. This result seems to be new.

A.1. The Homfly skein module. The Homfly skein module of an oriented 3-manifold is generated over the ring $\mathbb{Z}\left[x^{ \pm 1}, y^{ \pm 1}\right]$ by isotopy classes of oriented links modulo the skein relation of the Homfly polynomial

$$
\left.x \searrow \searrow-x^{-1} \swarrow \searrow=y\right)(
$$

where the links $\left.(\measuredangle, \searrow,)_{(}\right)$differ only locally as shown in the diagrams. For technical reasons, we include the empty link $\varnothing$ in the definition of the Homfly skein module and relate it to the trivial knot $O$ by the equation $\left(x-x^{-1}\right) \varnothing=y O$.

In difference to the Conway skein module, the 3-manifold must be oriented for the definition of the Homfly skein module, and we need no order nor basepoints on $L$. Let $\Sigma$ be an oriented decomposed surface in the sense of Section 3. Define the ordered set $\hat{\mathscr{S}}$ of representatives of conjugacy classes of elements in $\pi_{1}(\Sigma)$ as in Section 3. Then $\hat{\mathscr{Y}}$-descending links are defined by forgetting the order and basepoints of the links in Definition 3.3. Define $\hat{\mathscr{S}}^{\circ}=\hat{\mathscr{S}} \backslash\{e\}$, where $e \in \pi_{1}(\Sigma)$ is the neutral element. For a link $L \subset \sum \times I$ we define $L^{\circ}$ as $L$ without the components that are homotopic to $e$. Let $n(L)$ be the number of components of $L \backslash L^{\circ}$. Let $\mathbb{Z}\left[t_{\hat{\varphi}^{\circ}}\right]$ (resp., $\mathbb{Z}\left[t_{\hat{\varphi}}\right]$ ) be a polynomial ring with indeterminates $t_{w}$ in one-to-one correspondence with elements $w \in \hat{\mathscr{S}}^{\circ}$ (resp., $w \in \hat{\mathscr{Y}}$ ). For a knot $K \subset \Sigma \times I$ the element $t_{K} \in \mathbb{Z}\left[t_{\hat{\varphi}}\right]$ is defined as $t_{K}=t_{w}$, where $K$ is homotopic to $w$. For a link $L$ the element $t_{L}$ is defined as the product of elements $t_{K}$ where $K$ runs over all components of $L$. By $t_{\varnothing}$ we mean $1 \in \mathbb{Z}\left[t_{\hat{\varphi}}\right]$. Then we have the following theorem.

THEOREM A.5. There exists a unique invariant,

$$
H(L) \in \mathbb{Z}\left[x^{ \pm 1}, y^{ \pm 1}\right] \otimes_{\mathbb{Z}} \mathbb{Z}\left[t_{\hat{\varphi} \circ}\right],
$$

of links $L$ in $\Sigma \times I$ that depends only on the class of $L$ in the Homfly skein module and satisfies

$$
H(L)=t_{L^{\circ}}\left(\frac{x-x^{-1}}{y}\right)^{n(L)} \quad \text { whenever } L \text { is } \hat{\mathscr{Y}} \text {-descending. }
$$


The link invariant $H$ is called the Homfly polynomial. As in the proof of Lemma 5.1 we see that (A.1) and (A.3) are sufficient to calculate $H(L)$ for every link. This argument is much simpler than the corresponding proof for the Conway polynomial in Sections 5 and 6. This implies the uniqueness stated in Theorem A.5. Under the condition that $H$ factors through the Homfly skein module, it can easily be shown that (A.3) is implied by the same equation for links $L$ with $n(L)=0$. This implies that the Homfly skein module of $\sum \times I$ is a quotient of $\mathbb{Z}\left[x^{ \pm 1}, y^{ \pm 1}\right] \otimes_{\mathbb{Z}} \mathbb{Z}\left[t_{\hat{y}^{\circ}}\right]$. We give a sketch of the proof of the existence of $H$ in the rest of this section. This will imply that the Homfly skein module of $\sum \times I$ is isomorphic to $\mathbb{Z}\left[x^{ \pm 1}, y^{ \pm 1}\right] \otimes_{\mathbb{Z}} \mathbb{Z}\left[t_{\hat{y}^{\circ}}\right]$.

For a group $G$, define $\mathscr{A}(G)=\mathscr{A}^{\mathrm{ob}}(G, 0)$ and $\bar{A}(G)=\bar{A}^{\mathrm{ob}}(G, 0)$ (see Definitions 7.1 and 7.2). In these definitions the order and the basepoints on chord diagrams and relations (Ord) and (Bas) are superfluous because $\sigma=0$. We identify the degree- 0 part $\mathscr{A}_{0}(G)$ of $\mathscr{A}(G)$ with the isomorphic polynomial ring $\mathbb{Q}\left[t_{\hat{y}}\right]$. The analogue of Corollary 7.4 is not true for $\sigma=0$ because $t_{e} t_{w} \neq 0$ for $w \in G$. There are two equivalent ways of solving this problem. One way is used in the following, and the second way is used in Subsection A.2 for the Kauffman polynomial. For a $G$-labeled chord diagram $D$, we define the element $\iota(D)$ by replacing each chord as shown in Figure A.4.

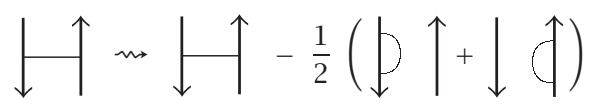

FIGURE A.4. The deframing map $\iota$.

The definition determines a linear map $\iota: \bar{A}(G) \rightarrow \mathscr{A}(G)$, such that $p \circ \iota=$ id where $p: \mathscr{A}(G) \rightarrow \bar{A}(G)$ denotes the canonical projection (compare [3, Exercise 3.16]). The map $\iota$ induces a map $\iota^{*}: \mathscr{A}(G)^{*} \rightarrow \bar{A}(G)^{*}$ called deframing projection. For $\sigma=0$ we denote the map $W_{\mathfrak{g} f}^{\mathrm{ob}}$ (see Proposition 7.3) also by $W_{\mathfrak{g} \mathfrak{l}}$. Define

$$
\bar{W}_{\mathfrak{g} \uparrow}: \bar{A}(G) \longrightarrow \mathbb{Q}\left[t_{\hat{g}}\right], \quad \bar{W}_{\mathfrak{g} \uparrow}=W_{\mathfrak{g} \uparrow} \circ \iota .
$$

The map $\bar{W}_{\mathrm{g} \text { r }}$ is called the weight system of the Homfly polynomial. Proceeding as in Section 8 , we extend $\bar{W}_{\mathfrak{g} \text { r }}$ to the completion $\hat{\mathscr{A}}(G)$ of $\overline{\mathscr{A}}(G)$ by

$$
\widehat{W}_{\mathfrak{g l}}(D)=\bar{W}_{\mathfrak{g l}}(D) h^{\operatorname{deg} D} \in \mathbb{Q}\left[t_{\hat{y}}\right][[h]] .
$$

For a link $L \subset \Sigma \times I$, let $\hat{H}(L) \in \mathbb{Q}\left[t_{\hat{y}}\right][[h]]$ be given by $\hat{H}(L)=\widehat{W}_{\mathfrak{g} \Gamma} \circ Z_{\Sigma \times I}(L)$, where $Z_{\Sigma \times I}$ denotes the universal Vassiliev invariant of links in $\Sigma \times I$. Denote the inclusion map $\mathbb{Q}\left[t_{\hat{y}}\right][[h]] \rightarrow \mathbb{Q}\left[t_{\hat{y}}\right]\left[\left[h, h^{-1}\right]\right]$ by $i$. We turn $\mathbb{Q}\left[t_{\hat{y}}\right]\left[\left[h, h^{-1}\right]\right]$ into a $\mathbb{Z}\left[x^{ \pm 1}, y^{ \pm 1}\right]$-module by

$$
x \cdot a=e^{t_{e} h / 2} a, \quad y \cdot a=\left(e^{h / 2}-e^{-h / 2}\right) a .
$$

With the notation from above we have the following lemma.

LEMmA A.6. The link invariant $i \circ \hat{H}$ induces a $\mathbb{Z}\left[x^{ \pm 1}, y^{ \pm 1}\right]$-linear map from the Homfly skein module to $\mathbb{Q}\left[t_{\hat{y}}\right]\left[\left[h, h^{-1}\right]\right]$ and satisfies

$$
\hat{H}(L) \equiv t_{L} \bmod h \quad \text { for every link } L .
$$


The proof of Lemma A.6 and the completion of the proof of Theorem A.5 are similar to the proofs in Section 8.

A.2. The Kauffman skein module. The Kauffman skein module of an oriented 3manifold is generated over the ring $\mathbb{Z}\left[x^{ \pm 1}, y^{ \pm 1}\right]$ by isotopy classes of framed oriented links modulo the relation that, reversing the orientation of a component, induces the identity map of the Kauffman skein module, and modulo the skein relations of the Kauffman polynomial,

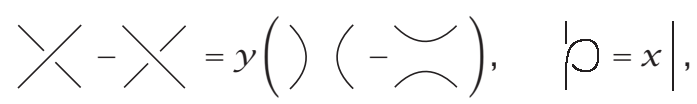

where the framed links $(Y, \lambda),(, \smile)$ ) and $(\rho, \mid)$ differ only locally as shown by the diagrams. In diagrams of framed links, the framing is assumed to be the so-called blackboard framing (the framing pointing to the reader). For technical reasons, we include the empty link $\varnothing$ in the definition of the Kauffman skein module and relate it to the trivial knot with 0 -framing $O$ by the equation $\left(x-x^{-1}+1\right) \varnothing=y O$.

Let $\Sigma$ be an oriented decomposed surface in the sense of Section 3. Define the ordered set $\hat{\mathscr{S}}$ of representatives of conjugacy classes of elements in $\pi_{1}(\Sigma)$ as in Section 3. Let $\hat{\mathscr{Y}}_{ \pm}=\left\{\min \left\{a, a^{-1}\right\} \mid a \in \hat{\mathscr{Y}}\right\}$. The set $\hat{\mathscr{Y}}_{ \pm}$is in one-to-one correspondence with homotopy classes of nonoriented knots. In this section, $\hat{\mathscr{S}}$-descending links are defined by forgetting the order and basepoints of the links in Definition 3.3 and equipping them with arbitrary framing.

For a diagram $L$ of a framed oriented link in $\Sigma \times I$, define the writhe of $L$ as $\mathrm{w}(L)=$ $k_{+}-k_{-}$, where $k_{+}$is the number of positive crossings and $k_{-}$is the number of negative crossings in the diagram. The writhe is an isotopy invariant of framed oriented links and of framed nonoriented knots.

Define $\hat{\mathscr{Y}}_{ \pm}^{\circ}=\hat{\mathscr{Y}}_{ \pm} \backslash\{e\}$, where $e \in \pi_{1}(\Sigma)$ is the neutral element. For a framed link $L$ we define $L^{\circ}, n(L)$, and $t_{L} \in \mathbb{Z}\left[t_{\hat{\mathscr{S}}_{ \pm}}\right]$in the same way as in Subsection A.1. Then we have the following theorem.

THEOREM A.7. There exists a unique invariant,

$$
F(L) \in \mathbb{Z}\left[x^{ \pm 1}, y^{ \pm 1}\right] \otimes_{\mathbb{Z}} \mathbb{Z}\left[t_{\hat{\varphi}_{ \pm}^{\circ}}\right],
$$

of framed links $L$ in $\Sigma \times I$ that depends only on the class of $L$ in the Kauffman skein module and satisfies

$$
F(L)=t_{L^{\circ}}\left(\frac{x-x^{-1}}{y}+1\right)^{n(L)} x^{\mathrm{w}(L)}, \quad \text { whenever } L \text { is } \hat{\mathscr{S}}_{ \pm} \text {-descending } .
$$

The link invariant $F$ is called the Kauffman polynomial. The polynomial $x^{-w(L)} F(L)$ is defined for each framed oriented link $L$ and does not depend on the framing of $L$, 
and hence is an isotopy invariant of oriented links. As in the proof of Lemma 5.1 we see that the conditions in Theorem A.7 are sufficient to calculate $F(L)$ for every link. This implies the uniqueness stated in Theorem A.7. We give a sketch of the proof of the existence of $F$ in the rest of this section. This will also imply that the Kauffman skein module of $\Sigma \times I$ is isomorphic to $\mathbb{Z}\left[x^{ \pm 1}, y^{ \pm 1}\right] \otimes_{\mathbb{Z}} \mathbb{Z}\left[t_{\hat{y}_{ \pm}^{\circ}}\right]$.

We say that a circle is locally oriented if it is decomposed into a finite number of oriented intervals. Let $C_{\text {loc }}(G)$ be the $\mathbb{Q}$-vector space generated by disjoint unions of locally oriented circles with a finite number of distinct points on oriented parts of the circles labeled by the elements of $G$ modulo homeomorphisms of these diagrams and the following relations:

(Rep):

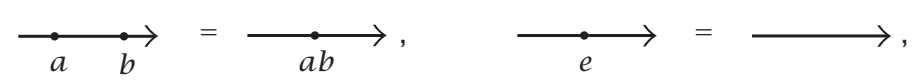

(Ori):

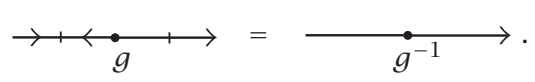

Here and in Figure A.5, we represent elements of $C_{\text {loc }}(G)$ graphically by formal linear combinations of parts of pictures of immersions of labeled, locally oriented circles. The points where the local orientation changes are marked by the symbol |.

Recall the definition of the vector space $\mathscr{A}(G)=\mathscr{A}^{\mathrm{ob}}(G, 0)$ (see Definition 7.1). Define a map $\beta_{1}: \mathscr{A}(G) \rightarrow C_{\text {loc }}(G)$ by replacing each chord as shown in Figure A.5 (it is easy to see that $\beta_{1}$ is well defined).

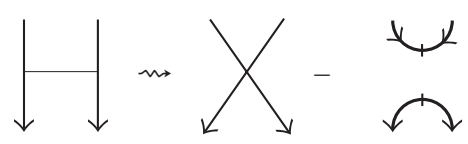

Figure A.5. The map $\beta_{1}$.

Using relations (Rep) and (Ori), we can replace each element of $C_{\text {loc }}(G)$ by a linear combination of disjoint unions of oriented circles labeled by a single element of $G$. Again by relations (Rep) and (Ori), oriented circles with labels $g, h g h^{-1}$, and $g^{-1}$ define the same element of $C_{\text {loc }}(G)$. A map $\beta_{2}: C_{\text {loc }}(G) \rightarrow \mathbb{Q}\left[t_{\hat{y}_{+}}\right]$is defined by replacing oriented circles with a label $g \in \hat{\mathscr{Y}}_{ \pm}$by $t_{g}$. It is easy to see that $\beta_{2}$ is well defined. We define the linear map

$$
W_{\text {osp }}: \mathscr{A}(G) \longrightarrow \mathbb{Q}\left[t_{\hat{9}_{ \pm}}\right], \quad W_{\text {osp }}=\beta_{2} \circ \beta_{1}
$$


The map $W_{\text {osp }}$ is called the weight system of the Kauffman polynomial. Proceeding as in Section 8 , we extend $W_{\text {osp }}$ to the completion $\tilde{A}(G)$ of $\mathscr{A}(G)$ by

$$
\widetilde{W}_{\text {osp }}(D)=W_{\text {osp }}(D) h^{\operatorname{deg} D} \in \mathbb{Q}\left[t_{\hat{y}_{ \pm}}\right][[h]] .
$$

For a framed oriented link $L \subset \sum \times I$ let $\widetilde{F}(L) \in \mathbb{Q}\left[t_{\hat{g}}\right][[h]]$ be given by

$$
\widetilde{F}(L)=\widetilde{W}_{\text {osp }} \circ Z_{\Sigma \times I}^{f}(L),
$$

where $Z_{\Sigma \times I}^{f}$ denotes the universal Vassiliev invariant of framed oriented links in $\Sigma \times I$. The invariant $Z_{\Sigma \times I}^{f}$ is defined by the same formulas as $Z_{\Sigma \times I}$ (see $[4,14,16]$ ). As in Subsection A.1 we denote the inclusion map $\mathbb{Q}\left[t_{\hat{y}_{ \pm}}\right][[h]] \rightarrow \mathbb{Q}\left[t_{\hat{\varphi}_{ \pm}}\right]\left[\left[h, h^{-1}\right]\right]$ by $i$. This time, we turn $\mathbb{Q}\left[t_{\hat{y}_{ \pm}}\right]\left[\left[h, h^{-1}\right]\right]$ into a $\mathbb{Z}\left[x^{ \pm 1}, y^{ \pm 1}\right]$-module by

$$
x \cdot a=e^{\left(t_{e}-1\right) h / 2} a, \quad y \cdot a=\left(e^{h / 2}-e^{-h / 2}\right) a .
$$

With the notation from above we have the following lemma (cf. [13]).

LEMmA A.8. The invariant $i \circ \tilde{F}$ of framed oriented links induces $a \mathbb{Z}\left[x^{ \pm 1}, y^{ \pm 1}\right]$-linear map from the Kauffman skein module to $\mathbb{Q}\left[t_{\hat{\varphi}_{ \pm}}\right]\left[\left[h, h^{-1}\right]\right]$ and satisfies

$$
\widetilde{F}(L) \equiv t_{L} \bmod h \text { for every link } L \text {. }
$$

SKETCH OF PROOF. Using $\left.W_{\text {osp }}(\lrcorner\right)=-W_{\text {osp }}(\longleftarrow)$ and the analogous dependence of $Z_{\Sigma \times I}^{f}$ on orientations we see that $\widetilde{F}(L)$ does not depend on the orientation of $L$.

Using $W_{\text {osp }}(\hookrightarrow)=\left(t_{e}-1\right) W_{\text {osp }}(\longrightarrow)$ and the explicit description of $Z_{\Sigma \times I}^{f}$, it is easy to see that

$$
\widetilde{F}(\bigcirc)=e^{\left(t_{e}-1\right) h / 2} \widetilde{F}(\mid) .
$$

The property $\widetilde{F}(L) \equiv t_{L} \bmod h$ follows directly from the analogous property of the degree- 0 part of $\tilde{Z}_{\Sigma \times I}^{f}$ and the definition of $W_{\text {osp }}$. Therefore, it remains only to show that (A.20) holds with $\kappa=1, \tilde{y}=e^{h / 2}-e^{-h / 2}$, and

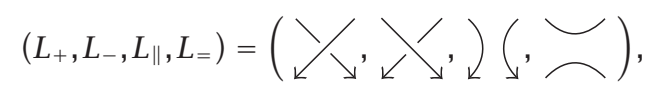

where the link $L_{=}$has arbitrary orientation,

$$
\tilde{F}\left(L_{+}\right)-\tilde{F}\left(L_{-}\right)=\tilde{y}\left(\tilde{F}\left(L_{\|}\right)-\kappa \tilde{F}\left(L_{=}\right)\right)
$$

For the following computation we extend $\widetilde{W}_{\text {osp }}$ to locally oriented labeled chord diagrams in the unique way that respects relation (Ori); we do not indicate the local 
orientation in the picture if it is not of importance:

$$
\begin{aligned}
& \tilde{F}\left(L_{+}\right)-\tilde{F}\left(L_{-}\right)=\widetilde{W}_{\text {osp }}(\exp (\downarrow \downarrow / 2) \circ \times)-\widetilde{W}_{\text {osp }}(\exp (-\downarrow \downarrow / 2) \circ \times) \\
& =\sum_{n=0}^{\infty} \widetilde{W}_{\text {osp }}\left(\left((X-\asymp)^{n}-(\asymp-X)^{n}\right) \circ X\right) \frac{h^{n}}{2^{n} n !} \\
& =\sum_{n=0}^{\infty} \widetilde{W}_{\text {osp }}\left(( X ^ { n } - ) \left(+()(-\asymp)^{n}-(-X)^{n}\right.\right. \\
& \left.\left.+(-)()^{n}-(\asymp-)()^{n}\right) \circ \times\right) \frac{h^{n}}{2^{n} n !} \\
& =\tilde{y} \tilde{F}\left(L_{\|}\right)+\sum_{n=0}^{\infty} \widetilde{W}_{\text {osp }}\left(( - ) \left(+()(-\smile)^{n}\right.\right. \\
& \left.\left.+(-)()^{n}-(\asymp-)()^{n}\right) \circ \times\right) \frac{h^{n}}{2^{n} n !} \\
& =\tilde{y} \tilde{F}\left(L_{\|}\right)+\frac{1}{t_{e}} \sum_{n=0}^{\infty} \widetilde{W}_{\text {osp }}\left(( - ) \left(+()(-乙)^{n}+(-)()^{n}\right.\right. \\
& \left.\left.-(\asymp-)()^{n}\right) \circ こ\right) \frac{h^{n}}{2^{n} n !} \\
& =\tilde{y} \tilde{F}\left(L_{\|}\right)+\frac{-e^{h / 2}+e^{\left(1-t_{e}\right) h / 2}+e^{-h / 2}-e^{\left(t_{e}-1\right) h / 2}}{t_{e}} \widetilde{W}_{\text {osp }}(\asymp) \\
& =\tilde{y}\left(\widetilde{F}\left(L_{\|}\right)-\frac{\left[t_{e}-1\right]_{e^{h / 2}}+1}{t_{e}} \widetilde{W}_{\text {osp }}(\asymp)\right),
\end{aligned}
$$

where in the last equation we used the notation

$$
\left[t_{e}-1\right]_{e^{h / 2}}=\frac{e^{\left(t_{e}-1\right) h / 2}-e^{-\left(t_{e}-1\right) h / 2}}{e^{h / 2}-e^{-h / 2}} .
$$

Let $O$ be the trivial knot with 0 -framing. It is easy to see that we have

$$
\widetilde{W}_{\text {osp }}\left(\frac{\sqrt{v^{1 / 2}}}{\sqrt{v^{1 / 2}}}\right)=\frac{\tilde{F}(O)}{t_{e}} \widetilde{W}_{\text {osp }}(\longleftarrow),
$$

where $v=Z_{f}(O)$. We have $\widetilde{F}(O) \equiv t_{e} \bmod h$ which implies that $\widetilde{F}(O)$ is invertible in $\mathbb{Q}\left[t_{\hat{y}_{ \pm}}\right]\left[t_{e}^{-1}\right][[h]]$. The above computations show that (A.20) holds with

$$
\kappa=\frac{\left(\left[t_{e}-1\right]_{\left.e^{h / 2}+1\right)}\right.}{\widetilde{F}(O)} \in \mathbb{Q}\left[t_{\hat{y}_{ \pm}}\right]\left[t_{e}^{-1}\right][[h]]
$$

Applying (A.20) to the link diagrams of Figure A.6 and using (A.18), we obtain

$$
\widetilde{F}\left(O^{2}\right)=\left(\left[t_{e}-1\right]_{e^{h / 2}}+\kappa\right) \tilde{F}(O)
$$


It is easy to see that $\widetilde{F}\left(O^{2}\right)=\widetilde{F}(O)^{2}$. With $\kappa$ as in (A.24), equation (A.25) has a unique solution satisfying $\tilde{F}(O) \equiv t_{e} \bmod h$, namely, $\widetilde{F}(O)=\left[t_{e}-1\right]_{e^{h / 2}}+1$. This implies that $\kappa=1$, which completes the proof.

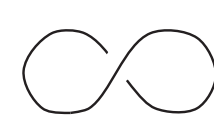

$\mathrm{O}_{1}$

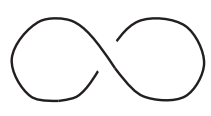

$O_{-1}$

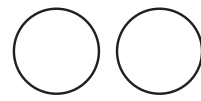

$\mathrm{O}^{2}$

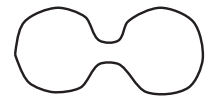

$O$

FIGURE A.6. Link diagrams used to determine $\tilde{F}(O)$.

Theorem A.7 follows from Lemma A.8 in a similar way as Theorem 4.1 follows from Lemma 8.1 (see Section 8).

ACKNOWLEDGmeNTS. I would like to thank C. Riedtmann and C. Kassel for helpful remarks. I thank the German Academic Exchange Service and the Schweizerischer National fonds for financial support. Research at Mathematical Sciences Research Institute (MSRI) is supported in part by National Science Foundation (NSF) grant DMS9701755.

\section{REFERENCES}

[1] J. W. Alexander, Topological invariants of knots and links, Trans. Amer. Math. Soc. 30 (1928), no. 2, 275-306.

[2] J. E. Andersen, J. Mattes, and N. Reshetikhin, Quantization of the algebra of chord diagrams, Math. Proc. Cambridge Philos. Soc. 124 (1998), no. 3, 451-467.

[3] D. Bar-Natan, On the Vassiliev knot invariants, Topology 34 (1995), no. 2, 423-472.

[4] _ Non-associative tangles, Geometric Topology proceedings of the Georgia International Topology Conference (Athens, GA, 1993) (W. H. Kazez, ed.), AMS/IP Stud. Adv. Math., vol. 2.1, American Mathematical Society, Rhode Island, 1997, pp. 139183.

[5] D. Bar-Natan and S. Garoufalidis, On the Melvin-Morton-Rozansky conjecture, Invent. Math. 125 (1996), no. 1, 103-133.

[6] R. C. Blanchfield, Intersection theory of manifolds with operators with applications to knot theory, Ann. of Math. (2) 65 (1957), 340-356.

[7] J. H. Conway, An enumeration of knots and links, and some of their algebraic properties, Computational Problems in Abstract Algebra (Proc. Conf., Oxford, 1967), Pergamon, Oxford, 1970, pp. 329-358.

[8] P. Freyd, D. Yetter, J. Hoste, W. B. R. Lickorish, K. Millett, and A. Ocneanu, A new polynomial invariant of knots and links, Bull. Amer. Math. Soc. (N.S.) 12 (1985), no. 2, 239-246.

[9] R. Hartley and A. Kawauchi, Polynomials of amphicheiral knots, Math. Ann. 243 (1979), no. $1,63-70$.

[10] V. F. R. Jones, A polynomial invariant for knots via von Neumann algebras, Bull. Amer. Math. Soc. (N.S.) 12 (1985), no. 1, 103-111.

[11] L. H. Kauffman, An invariant of regular isotopy, Trans. Amer. Math. Soc. 318 (1990), no. 2, 417-471.

[12] A. Kawauchi, A Survey of Knot Theory, Birkhäuser Verlag, Basel, 1996, translated and revised from the 1990 Japanese original by the author.

[13] T. Q. T. Le and J. Murakami, Kontsevich's integral for the Kauffman polynomial, Nagoya Math. J. 142 (1996), 39-65. 
[14] _ The universal Vassiliev-Kontsevich invariant for framed oriented links, Compositio Math. 102 (1996), no. 1, 41-64.

[15] J. Lieberum, Universal Vassiliev invariants of links in coverings of 3-manifolds, preprint, 2001, http://arxiv.org/abs/math/math.QA0105019.

[16] _ Invariants de Vassiliev pour les entrelacs dans $S^{3}$ et dans les variétés de dimension trois, Thèse de doctorat, Université Louis Pasteur (Strasbourg I), 1998, IRMA preprint.

[17] G. R. Livesay, Involutions with two fixed points on the three-sphere, Ann. of Math. (2) 78 (1963), 582-593.

[18] J. H. Przytycki, Skein module of links in a handlebody, Topology '90 (Columbus, OH, 1990), Ohio State Univ. Math. Res. Inst. Publ., vol. 1, de Gruyter, Berlin, 1992, pp. 315-342.

[19] _ Algebraic topology based on knots: an introduction, KNOTS '96 (Tokyo), World Sci. Publishing, New Jersey, 1997, pp. 279-297.

[20] J. H. Rubinstein, Heegaard splittings and a theorem of Livesay, Proc. Amer. Math. Soc. 60 (1976), 317-320.

[21] P. A. Smith, Fixed Points of Periodic Transformations, vol. 27, Amer. Math. Soc. Colloquium Publications, New York, 1942, Appendix B in S. Lefschetz, Algebraic Topology.

[22] A. Vaintrob, Melvin-Morton conjecture and primitive Feynman diagrams, Internat. J. Math. 8 (1997), no. 4, 537-553.

[23] V. A. Vassiliev, Cohomology of knot spaces, Theory of singularities and its applications (V. I. Arnold, ed.), Amer. Math. Soc., Rhode Island, 1990, pp. 23-69.

[24] P. Vogel, Invariants de Vassiliev des nouds (d'après D. Bar-Natan, M. Kontsevich et V. A. Vassiliev), Astérisque (1993), no. 216, 213-232 (French).

JENS LIEBERUM: MATHEMATISCHES INSTITUT, UNIVERSITÄT BASEL, RHEINSPRUNG 21, CH-4051 BASEL, SWITZERLAND

E-mail address: 1 i eberum@math . unibas.ch 


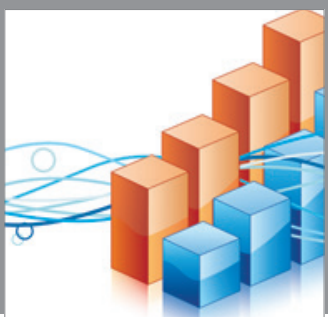

Advances in

Operations Research

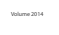

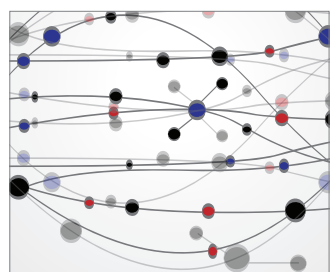

\section{The Scientific} World Journal
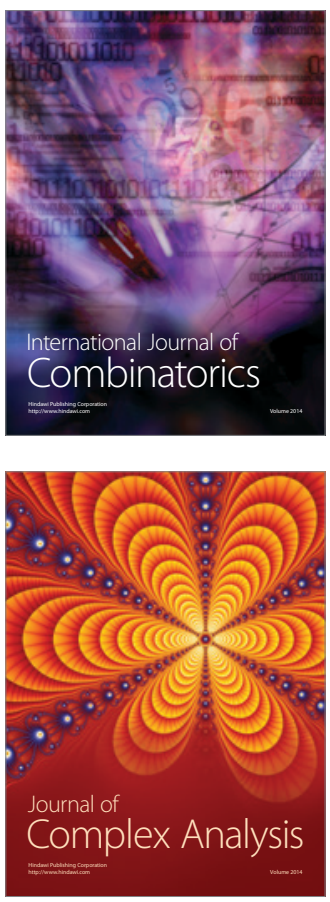

International Journal of

Mathematics and

Mathematical

Sciences
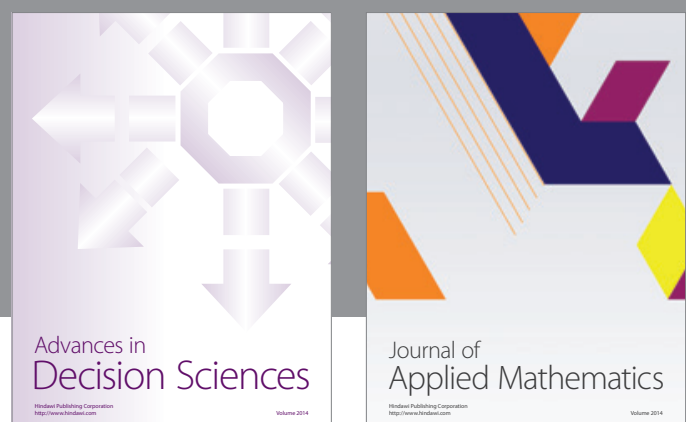

Journal of

Applied Mathematics
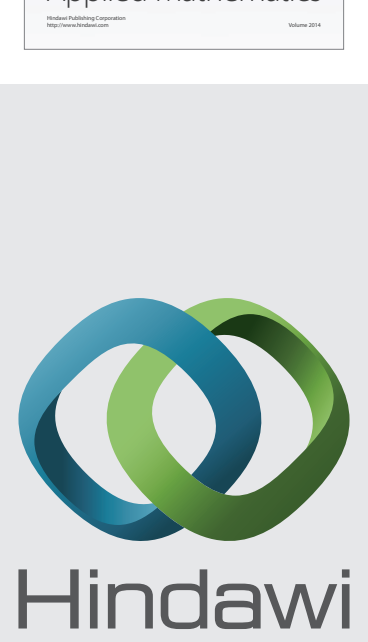

Submit your manuscripts at http://www.hindawi.com
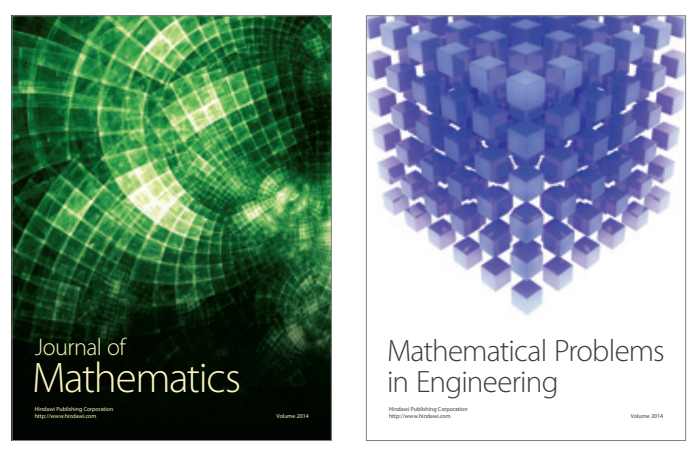

Mathematical Problems in Engineering
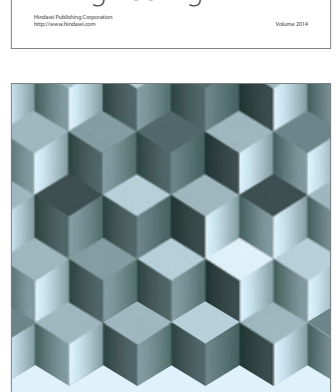

Journal of

Function Spaces
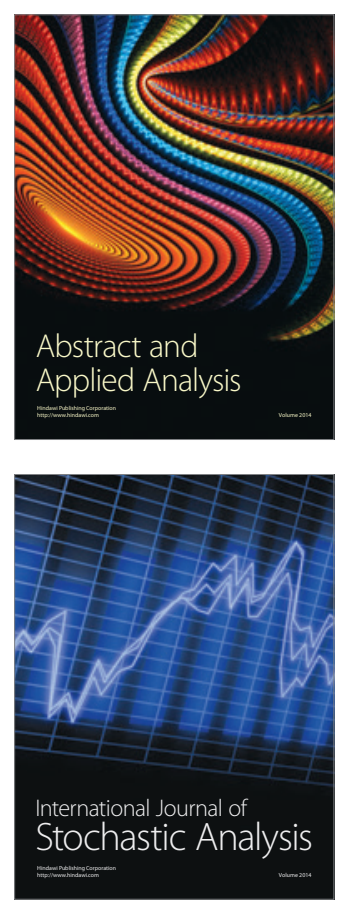

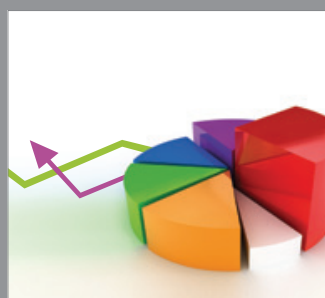

ournal of

Probability and Statistics

Promensencen
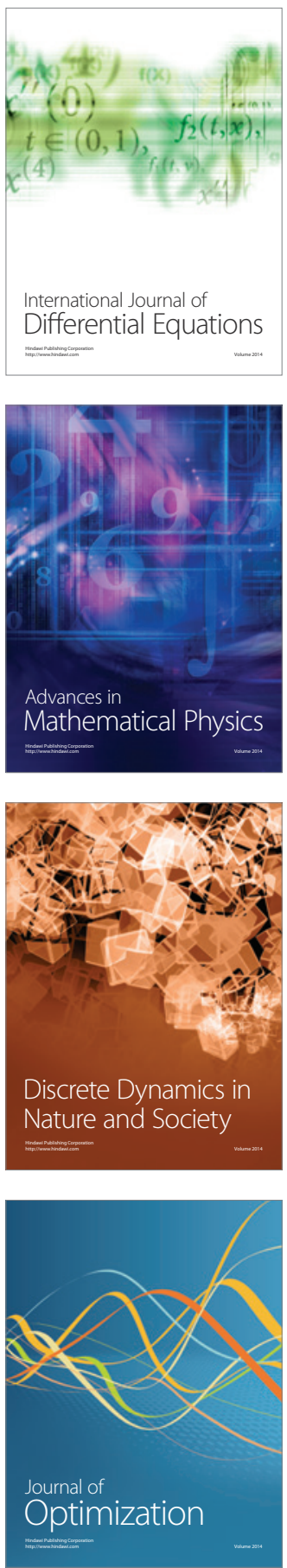